\title{
Radio Resource Allocation Improvements in CRSN for Smart Grid: A Survey
}

\author{
Emmanuel Ogbodo ${ }^{1}$, David Dorrell ${ }^{1 *}$ and Adnan Abu-Mahfouz ${ }^{2}$ \\ 1 Discipline of Electrical, Electronic and Computer Engineering, University of KwaZulu-Natal, Durban, South Africa; \\ email: ogbodoeu@ieee.org and dorrelld@ukzn.ac.za \\ 2 Council for Scientific and Industrial Research (CSIR) Pretoria, South Africa; email: a.abumahfouz@ieee.org \\ * Correspondence: dorrelld@ukzn.ac.za and ogbodoeu@ieee.org
}

\begin{abstract}
A cognitive radio sensor network (CRSN) based Smart Grid (SG) is a new paradigm for a modern SG. It is totally different from the traditional power grid and also different from the conventional SG that uses a static resource allocation technique to allocate resources to sensor nodes and communication devices in the SG network. Due to the challenges associated with competitive sensor nodes and communication devices in accessing and utilizing radio resources, the need for dynamic radio resource allocation (RRA) has been proposed as a solution for allocating radio resources to sensor nodes in a CRSN based smart grid ecosystem (network). These challenges include energy/power constraints, poor quality of service (QoS), interference, delay, spectrum efficiency issues, and excessive spectrum hand-offs. Hence, the optimization of resource allocation criteria, such as energy efficiency, throughput maximization, QoS guarantee, fairness, priority, interference mitigation/avoidance, etc., will go a long way in addressing the problems of RRA in a CRSN based SG. Consequently, this work explores RRA in CRSNs for SGs. Various resource allocation schemes, as well as its architecture in a CRSN for SG environment, are presented. The work reported in this paper introduces a model called the "guaranteed network connectivity channel allocation" for throughput maximization (GNC-TM) and optimal spectrum band determination in RRA for improved throughput criteria in CRSNs for SGs. The results show that the model outperforms the existing protocol in terms of throughput and error probability. Finally, the contribution to knowledge and future research direction, such as energy efficiency and hybrid energy harvesting schemes are highlighted.
\end{abstract}

Keywords: Adaptive Modulation, TVWS, CRSN, RRA, Smart Grid, Distributed Heterogeneous Clustered (DHC), Dynamic radio.

\section{Nomenclature}

$\begin{array}{ll}\text { CPG } & \text { Central power generation } \\ \text { CR } & \text { Cognitive radio } \\ \text { CRN } & \text { Cognitive radio network } \\ \text { CRSN } & \text { Cognitive Radio Sensor Network } \\ \text { CSMA/CA } & \text { Carrier sense multiple access and collision avoidance } \\ \text { DA } & \text { Distribution automation } \\ \text { DER } & \text { Distributed Energy Resources } \\ \text { DREG } & \text { Distributed renewable energy generation } \\ \text { DES } & \text { Discrete event simulation } \\ \text { EV } & \text { Electric Vehicle } \\ \text { EMC } & \text { Electromagnetic Comparability } \\ \text { EMI } & \text { Electromagnetic Interference } \\ \text { GSM } & \text { Global System for mobile communication } \\ \text { HAN } & \text { Home Area Network } \\ \text { IoT } & \text { Internet of Things }\end{array}$




\begin{tabular}{|c|c|c|}
\hline 48 & IPO & independent power operator \\
\hline 49 & ISM & Industrial scientific and medical \\
\hline 50 & LPWAN & Low power wide area network \\
\hline 51 & MAC & Medium access control \\
\hline 52 & MDMS & Meter data management system \\
\hline 53 & NAN & Neighborhood area network \\
\hline 54 & NETSIM & Network simulator \\
\hline 55 & NIST & National Institute of Standards and Technology \\
\hline 56 & PHY & Physical layer \\
\hline 57 & PLC & Power line communication \\
\hline 58 & PMU & Phasor management unit \\
\hline 59 & PU & Primary user \\
\hline 60 & QoS & Quality of Service \\
\hline 61 & RRA & Radio resource allocation \\
\hline 62 & SCADA & Supervisory control and data acquisition \\
\hline 63 & SU & Secondary user \\
\hline 64 & TV & Television \\
\hline 65 & TVWS & TV white space \\
\hline 66 & CVWS & Cellular white space \\
\hline 67 & UDP & User datagram protocol \\
\hline 68 & UHF & Ultra high frequency \\
\hline 69 & VHF & Very high frequency \\
\hline 70 & UMTS & Universal Mobile Telecommunications Service \\
\hline 71 & WAN & Wide area network \\
\hline 72 & WIFI & Wireless fidelity \\
\hline 73 & WiMAX & Worldwide Interoperability for Microwave Access \\
\hline 74 & WLAN & Wireless local area network \\
\hline 75 & WSN & Wireless Sensor Network \\
\hline
\end{tabular}

\section{Introduction}

\subsection{Background}

Traditional power grids use a top-down layer approach where the communication flow is only in one direction from the utility to the consumers. A Smart Grid (SG) has a bidirectional communication and information flow between utility and consumer. There are several communication technologies such as wired or wireless technologies, which can be used to realize the bidirectional communication in SG. Wireless communication is a good communication technology option to drive SG due to the extensive coverage area required in SG. However, the wireless channels in the wireless communication undergo a wide range of impediments such as fading, path loss and interference caused by other wireless devices operating in the Industrial, Scientific, and Medical (ISM) free band. There is also spectrum limitation and spectrum efficiency issues due to the high cost of acquiring a spectrum channel and poor spectrum utilization (only about $15 \%$ of the allocated spectrum is utilized).

To this end, to address the impairments and spectrum issues, a CRSN which is a combination of CR and WSN is proposed as adequate communication technologies in SG. The CRSN will enable Power Generation, Transmission, Distribution, Utilities, and Customers to transfer, monitor, predict, control and manage energy usage effectively and in a cost-efficient manner. CRSN can leverage television white space (TVWS) for SG communication. TVWS has been recommended in high-speed communication technology for balancing energy production and consumption in SG [1]. 
The realization of CRSN for the smart grid mainly requires efficient RRA strategies to manage the Dynamic Spectrum Access (DSA) of cognitive radio sensor nodes in harsh smart grid propagation environments. To meet the requirements of data rate and power constraints of the CRSN users, as well as to avoid interference, researchers all over the world are working hard to develop radio RRA scheme to effectively manage radio resources. CRSN has the potential advantages of reconfigurability and DSA capabilities; to exploit these potential advantages of CRSN, a dynamic efficient RRA among the sensor nodes is essential.

A traditional electricity grid has shortfalls in terms of effective monitoring, predicting, control and management of the energy in a cost efficient manner. This can fall short of the expectation of a modern electricity market.

\subsection{SG Architectural Framework}

An SG has functional subsystems that interact independently or cooperatively as shown in the framework in Figure 1. This framework shows the components or subsystems that make up the SG. The functional subsystems are as follows:

\subsubsection{Power system layer}

This comprises of the central power generation (CPG), distributed renewable energy generation (DREG), transmission, and distribution by utilities, with power supplied to the consumers.

\subsubsection{Control layer}

This subsystem consists of control systems such as the meter data management systems (MDMSs), supervisory control and data acquisition (SCADA), algorithmic applications and the MDMS server at the control/substation/data centre. It enables the control and management functions in the SG.

\subsubsection{Security layer}

This involves cybersecurity and provides data confidentiality, integrity, authentication, and availability for safe electricity distribution and counter-theft. Industrial Control Systems (ICS) such as SG comprising actuator and sensor networks are vulnerable to attacks that could lead to a devastating impact on the entire SG [2]. Hence, the security layer handles the vulnerability in the SG ecosystem.

(1)

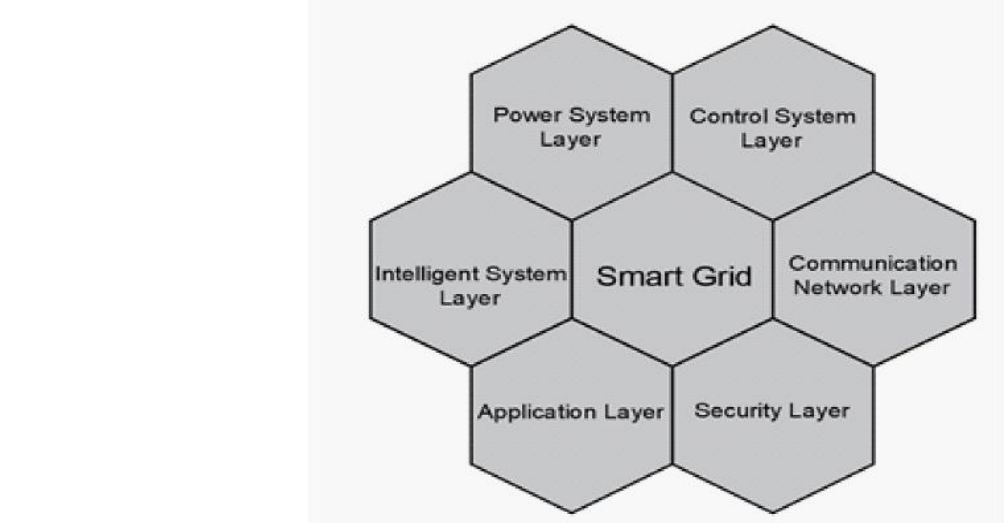

Figure 1. Smart Grid Architectural Framework

\subsubsection{Application layer}

This delivers numerous SG applications such as DER, AMI, DRM, and so on, to customers as well as utilities. 


\subsubsection{Intelligent layer}

This consists of intelligent electronic devices (IEDs) and sensors for monitoring and control in SCADA, MDMSs, and communications.

\subsubsection{Communication network layer}

This allows bi-directional communications in an SG. It consists of wireless cellular communication (GSM, GPRS, LTE, UMTS, EDGE, and so on), WiMAX, power line communication (PLC), Digital Subscriber Line (DSL), Ethernet, Fiber optics, machine-to-machine communication (M2M) such as WIFI, WSN, CRSN, ZigBee, Bluetooth, Low power wide area (LPWA) devices, and so on [3].

A critical analysis of the framework will deduce that the communication network layer is the key enabler for the delivery of information/data about the power system, control, applications, and so on. However, the aspect of M2M communication is of the utmost importance in an SG implementation. This paper considers RRA in CRSNs based M2M communication for a SG. This is because a CRSN has numerous advantages than WSN and CRN as shown in Table 1, which emphasizes the comparative framework that characterizes WSN, CRSN, and CRN based on some features or metrics. Another emerging area in M2M communication that is also advantageous for a SG and internet of things (IoT) implementation is the LPWA devices. Though LPWA is not the focus of this work.

\subsection{Challenges of CRSN in $S G$}

There are challenges associated with a CRSN, which can adversely affect adequate resource allocation within a CRSN in an SG. There are described below:

\subsubsection{Intermittent channel availability for a SU network}

PU activities can cause intermittent channel availability to an SU network. This is because whenever a PU arrives to use the channel, the SU relinquishes it. When this occurs too frequently it mars the correct communication of the CRSNs for adequate resource allocation.

\subsubsection{High bit error probability of detection of the PU}

When the SU has a high probability of an error in the detection of the presence of a PU, it will lead to false detection which affects the SU network negatively or causes harmful interference in the PU network. Hence, this issue is a research challenge that requires the mitigation of the high probability of an error in detection by the SU.

\subsubsection{The problem of limited spectrum holes due to PU activities}

Frequent PU activities will lead to fewer spectrum holes. There can impact adversely on the performance of the SU network. Creating multiple spectrum channels for the SU will lead to more spectrum holes which will help to avert the problem. Part of this challenge is addressed in Section 4.2,

where further analysis was carried out in order to establish a suitable spectrum band with more white space for CRSNs in a SG.

\subsubsection{Adequate protocol for CRSN in an SG}

Protocols that are suitable for a CRSN in a SG are in their infancy since a CRSN is a new paradigm and its protocol is quite different from that of a conventional wireless system which has higher computational complexity. 
Table 1. Comparative framework for WSN, CRSN, and CRN

\begin{tabular}{|c|c|c|c|}
\hline Features/Metric & WSN & CRSN & CRN \\
\hline Channel access & Fixed channel access & Multiple/dynamic & Multiple/dynamic \\
\hline $\begin{array}{l}\text { Organizing and self- } \\
\text { healing }\end{array}$ & Moderate & Very high & Very high \\
\hline $\begin{array}{l}\text { Interference } \\
\text { avoidance }\end{array}$ & Low & High & High \\
\hline Network topologies & $\begin{array}{l}\text { Star, Cluster-tree, } \\
\text { and Mesh }\end{array}$ & $\begin{array}{l}\text { Star, Mesh, Cluster, } \\
\text { Hierarchical, Mobile } \\
\text { Ad Hoc, and } \\
\text { Distributed } \\
\text { Heterogeneous } \\
\text { Clustered (DHC) }\end{array}$ & $\begin{array}{l}\text { Star, Mesh, } \\
\text { Hierarchical, Mobile } \\
\text { Ad Hoc (MANET) }\end{array}$ \\
\hline $\begin{array}{l}\text { Communication } \\
\text { protocol stack }\end{array}$ & $\begin{array}{l}\text { Physical, Data link, } \\
\text { Network, and } \\
\text { application layer }\end{array}$ & $\begin{array}{l}\text { Physical, Data link, } \\
\text { Network, Transport, } \\
\text { and application layer }\end{array}$ & $\begin{array}{l}\text { Physical, Data link, } \\
\text { Network, Transport, } \\
\text { and application layer }\end{array}$ \\
\hline $\begin{array}{l}\text { Data } \\
\text { centricity/unification }\end{array}$ & Highly supported & Highly supported & Less supported \\
\hline $\begin{array}{l}\text { Energy } \\
\text { conservation/harvestin } \\
\text { g }\end{array}$ & High & High & Medium \\
\hline $\begin{array}{l}\text { Efficient energy } \\
\text { consumption }\end{array}$ & $\begin{array}{l}\text { Low (More energy } \\
\text { waste) }\end{array}$ & $\begin{array}{l}\text { High (energy } \\
\text { efficiently used) }\end{array}$ & $\begin{array}{l}\text { High (energy } \\
\text { efficiently used) }\end{array}$ \\
\hline $\begin{array}{l}\text { Application specific } \\
\text { driven }\end{array}$ & Highly Supported & Highly supported & Less supported \\
\hline scalability & $\begin{array}{l}\text { Large scale (supports } \\
\text { thousands of nodes) }\end{array}$ & $\begin{array}{l}\text { Large scale (supports } \\
\text { thousands of nodes) }\end{array}$ & $\begin{array}{l}\text { Medium scale } \\
\text { (supports hundreds of } \\
\text { nodes) }\end{array}$ \\
\hline Coverage range & Short range & $\begin{array}{l}\text { Short to medium } \\
\text { range }\end{array}$ & Long range \\
\hline Environment sensing & $\begin{array}{l}\text { Sense any target } \\
\text { phenomenon }\end{array}$ & $\begin{array}{l}\text { Sense any target } \\
\text { phenomenon and } \\
\text { radio properties }\end{array}$ & $\begin{array}{l}\text { Sense mainly radio } \\
\text { properties (spectrum } \\
\text { channels, modulation, } \\
\text { power control) }\end{array}$ \\
\hline $\begin{array}{l}\text { Computational } \\
\text { complexity }\end{array}$ & Low & Medium & High \\
\hline
\end{tabular}

173 It is different from a conventional WSN which has lower complexity, but the computational complexity 174 for a CRSN is of medium complexity; hence, it requires a protocol that matches its functionalities which 175 will help to realize adequate resource allocation in an SG communication system. Its protocol is unique 176 due to the dynamic multiple channel access, whereas the protocol for conventional wireless has fixed 177 channel access.

178 1.3.5. Problems of communication infrastructure in SG with regards to the requirements for SG deployment 
The communication equipment is susceptible to challenges associated in a SG environment. For example, power-frequency electromagnetic fields and radiofrequency (RF) noise exist in the SG environment due to corona and partial discharges, solid-state and substation switching devices, and circuit breaker switching, including commutating processes [4]. These can result in electromagnetic interference (EMI) issues which are known to cause interference and failure of electronic devices and communication infrastructure [4]. These disturbances and environmental changes negatively impact communications infrastructure and its operation.

Therefore, communications infrastructure needs to be strong enough to operate in harsh SG environments. The International Special Committee on Radio Interference (CISPR) investigated radio noise originating from high voltage (HV) power equipment and provided recommendations for reducing the radio noise generated in SGs [5]. Impulse noise has been investigated in HV substations including its influence on the performance of wireless channels and modulations [6]. EMI impacts SG wireless communication equipment and this was studied in [7]. Hence, it is necessary to define the appropriate compliance requirements in an SG to ensure the reliable performance of the wireless communications infrastructure.

To this end, the International electrotechnical commission (IEC) has enacted the following key immunity compliance requirements for use in SGs with regards to the communication network infrastructure:

- IEC 61850-3 - Part 3: General requirements for communication networks and systems for SG utility automation.

- IEEE 16.13-2003 - IEEE standard environmental and testing requirements for communications network infrastructure in SG Substations. Its

- IEEE 16.13.1-2013 - IEEE standard environmental and testing requirements for communication network infrastructure Installed in SG transmission and distribution facilities.

Consequently, RRA in CRSNs for other applications is different from the RRA in CRSNs for SG applications. That makes this survey quite different from other related surveys on CRSNs. Hence, RRA in CRSN for SG applications should be based on the following considerations:

- Consideration of key immunity compliance requirements for the CRSN in an SG as stated earlier.

- Appropriate resource allocation architecture to cope with the EMI in the SG environment.

- Consideration of appropriate electromagnetic comparability (EMC) for the CRSN to operate effectively in a varying EMI SG environment.

\subsection{Protocol Architecture for a CRSN in an SG}

The SG has applications in order to operate in the various SG communication layers such as HAN, NAN, and WAN. Hence, heterogeneous communication technologies are required for the delivery of SG application data. The tough SG environment caused by harmonics, power line disturbance, cochannel interference from grid instruments, and severe propagation conditions, impairs SG communication. Hence, conventional protocols are not suitable for SG communication because of the varying applications, heterogeneous communication requirement and unsteady nature of the SG environment. To address these challenges, the protocol architecture for CRSN based SG communication must be: 
(1) Application-Specific driven/Aware; and

(2) Cross-layer framework.

- Application-Specific driven/Aware: since SG applications are for specific grid needs, they cannot be regarded as general purpose applications. Hence, the protocol architecture should be designed to support the specific purpose of the SG application, i.e., the heterogeneous communication requirement. The protocol architecture for the application should be spectrum aware. This means that the application should have an interaction with the MAC protocol of the CRSN in the SG.

- Cross-layer framework: since the channel condition in a CRSN based SG changes dynamically, there is a need for the underlying protocol stack to interact and change the information/signal. Thus, the protocol architecture should be designed in such a way that the Physical, MAC, Routing, Transport, and Application protocol layers interact with each other for information exchange.

Other considerations of the protocol architecture for a CRSN based SG include consideration of the common attributes of the CRSN such as low power, limited complexity, and channel characteristics. Hence, these attributes should be included in the protocol architecture. This signifies that the protocol architecture in the CRSN based SG should be based on energy efficiency as well as being spectrum aware.

Furthermore, the protocol architecture may be designed to typify a particular RRA architecture, such as centralized, clustered, distributed, and DHC architecture respectively. The channel characteristics/energy efficiency and device connectivity are common in the MAC and Routing protocols. Thus, most concerns are in these protocol layers, which can be designed to interact with the application layer by implementing the protocol design with a cross-layer framework.

The notable protocol architecture characteristics based on MAC protocol for a CRSN in a SG are:

- CRB-MAC: this protocol was proposed in [8]. The nodes leverage an optimal transmission by using a wake and sleep schedule timer for detecting the PU activities. It goes to sleep when PU is actively using the channel, and resumes again at the expiration of the time. However, this protocol is a receiver-based MAC protocol and is energy efficient with a reduced delay. However, it is not based on a cross-layer framework.

- CSMA/CA MAC: this protocol was proposed in [9]. This is based on a cross-layer framework approach that incorporates the CSMA/CA MAC protocol with dynamic spectrum access (DSA) to assess the available channels. The advantages of this protocol include the supporting of application-specific driven application, addresses QoS requirements, has a reduced delay, and has optimal throughput.

The notable protocol architecture characteristics based on Routing Protocol for a CRSN in a SG are:

- Distributed control algorithm (DCA): this protocol was proposed in [10]. This protocol is based on a cross-layer framework that interacts jointly in optimizing the routing, MAC and physical layer protocol functions in a CRSN to avoid the tough propagation conditions in a SG. This includes QoS support for SG applications.

- RPL (routing protocol for low power and lossy networks) modification: this protocol modification was proposed in [11] for energy and spectrum efficiency in a CRSN at the SG utility. This protocol is based on a multi-layered framework approach, and has the following advantages: reliability and low latency routing support for large-scale CRSNs.

Based on the above, it can be seen that the existing protocol architecture for a CRSN in a SG are very few. None of the protocol architecture for a CRSN in a SG supports a cross-layer framework that cuts across the five entire protocol stacks. Hence, a reliable cross-layer framework approach that jointly interacts with the Physical, MAC, Routing, Transport, and Application layer protocols would be 
advantageous in CRSN based SG communication. The protocol should be energy efficient as well as spectrum-aware for optimal SG communication.

The remainder of this paper is structured as follows in the following sections: Related works are discussed. Description of the overview, functionalities, and unique characteristics of a CRSN in a SG are presented. The RRA in a CRSN for SG is presented. Performance analysis of RRA based on throughput improvement criteria in CRSN for SG is presented. Recommendations and future research directions are discussed. Finally, the survey article ends with conclusions.

The focus of this paper is to explore RRA in a CRSN based SG, thus leading to the following contributions in this survey:

- A comprehensive survey of RRA in a CRSN based SG is presented.

- The overview, functionalities and unique characteristics of a CRSN in a SG are discussed.

- An SG Architectural Framework, including a comparative framework for WSN, CRSN, and CRN, is exemplified.

- A guaranteed network connectivity channel allocation for throughput maximization (GNC-TM) in CRSNs for SGs.

- Optimal spectrum band determination in RRA for improved throughput criteria in order to establish suitable spectrum band operation in CRSNs for SGs.

- The protocol architecture for a CRSN in a SG is highlighted.

- Radio resources optimization criteria in a CRSN based SG are discussed in this survey.

- An RRA scheme in a CRSN based SG, including its architecture, is presented.

- Recommendations and future research directions regarding the RRA in a CRSN based SG are highlighted.

\subsection{Overview, functionalities, and unique characteristics of a CRSN in an SG}

\subsubsection{Overview of CRSN}

In a CRSN, there are two types of users: primary and secondary. Primary users (PUs) are the licensed (authorized) users, who have the license to operate in an allotted spectrum band so they can access the primary base station. Secondary users (SUs) or Cognitive Radio users (CRs) are unlicensed users without a spectrum license. CRs use the existing spectrum through opportunistic access without causing harmful interference to the PUs. CRs look for the available portion of the spectrum that is not in use, which is called a spectrum hole or White Space. The SUs can share the spectrum channels with the PUs by using one of the two methods known as overlay and underlay methods. In an overlay method, SUs can opportunistically access the PU spectrum channels only if the channels are completely unused by the PUs. Whereas, in the underlay method, the SUs can simultaneously access the PU channels even when the PUs are using the channels so long as the harmful interference caused to the PUs is below a predetermined threshold value.

However, there are problems associated with the two methods. For instance, in the overlay method, some wireless services, such as TV and cellular networks, the PU channels may be predominantly busy for a long time, resulting to no white space. Hence, the SUs may be unable to opportunistically access the spectrum channels since there is no white space available in the PU networks. On the other hand, the problem in the underlay method involves the inability of the SUs to opportunistically access channels in an area predominantly deployed with PUs. This is because more interference will be caused to closely located PUs, thereby making it difficult for the SUs to access these channels within a state of interference. Therefore, it is essential to solving these problems that are associated with the overlay and underlay methods in CRSNs. 
Therefore, this paper employs the overlay method in CRSNs and throughout this paper the overlay method is adopted. The SUs use the optimal available channel only if there is no PU operating in the licensed bands [12]. The problem of the inability of the SUs to access channels in overlay method has been addressed in previous work [13]. In the work, a channel fragmentation strategy is used in a (CFS)based Alamouti space-frequency block coded (SFBC) scheme to improve the performance of the SU networks.

\subsubsection{Functionalities of CRSN}

A CRSN has the following cognitive functionalities to enable the secondary users to have dynamic and opportunistic access to the spectrum holes [14]. These functionalities are spectrum sensing, spectrum decision, spectrum sharing, and spectrum mobility. These four main cognitive radio functionalities are required to determine the accurate communication parameters of SG communication and adjust to the dynamic radio environments [15].

\subsubsection{Spectrum Sensing}

Spectrum sensing is the process of discovering of the available spectrum bands and detection of the spectrum holes in the PUs [16]. Spectrum sensing operation is a very power-consuming function and poses great challenges for providing seamless communications in large-scale SG deployments. Therefore, some solutions need to be deployed to achieve viable CRSN based SG communications. Minimum hardware, for example using single radio, and less advanced spectrum sensing functionalities, can be used to lower the complexity level of the sensing operations and reduce energy consumption [12], [17]. Reducing the sensing durations to an appreciable level can be a good solution. There are various spectrum sensing techniques, such as energy detection, feature detection, matched filter, and interference temperature [15]. Using one or a combination of these methods can be achieved.

Generally, spectrum sensing comes with additional energy consumption. Hence, there is a trade-off between sensing accuracy and energy efficiency. Therefore, an optimized DSA is required in order to address the spectrum accuracy which involves the lowering of packet collisions and the ability of switching to the best available channel, including less contention delay and enhanced bandwidth.

Spectrum sensing faces the challenge of being very sensitive to the detection mechanism due to harsh environmental conditions such as multipath fading and environmental noise in a SG environment. However, an optimised DSA will help in addressing this.

\subsubsection{Spectrum Decision}

The spectrum decision process involves two steps: spectrum characterization and spectrum selection. These are the necessary steps for the characterization of the spectrum band in terms of the received signal strength, interference, power of transmission and energy efficiency, number of communication users, QoS, and security requirements of SG applications [18]. Therefore, providing a QoS-aware cognitive communication network is essential in order to choose the appropriate spectrum band to meet the specific requirements of CRSN based SG communications. This is part of the spectrum decision process. However, a SG system environment has a distributed nature, and interference from radio signals, as well as the network density and channel characteristics, vary over a wide geographical area. This limits obtaining optimal knowledge about the spectrum availability. Consequently, this problem poses challenges in making precise spectrum decisions and meeting QoS requirements of CRSN SG applications.

\subsubsection{Spectrum Sharing}


The spectrum sharing process involves the selection of the best channel and power allocation. Some of the functionalities are related to the main functionalities of medium access control (MAC) layer protocols. Hence, it can be incorporated into the MAC layer. However, there are challenges associated with efficient spectrum sharing which include time synchronization and distributed power allocation [19]. For instance, methods of controlling power are essential for the spectrum sharing process in large SGs. These can adapt to the radio environments and maximize the network life-time [20]. Precise time measurements and time synchronization are required for some SG applications, such as equipment fault diagnostics and phasor measurement monitoring applications.

An effective spectrum sharing technique helps to meet the QoS requirements in a CRSN SG by adaptively allocating communication network resources. The opportunistic dynamic spectrum access capability can be used to adjust the communication transmission parameters to lessen redundant power consumption of CR sensor nodes thereby preventing the performance degradation of CRSN based SG communications.

\subsubsection{Spectrum Mobility}

Spectrum mobility, which is also called spectrum handoff, is used to mitigate the interference caused by SG communication infrastructure. Spectrum handoff occurs when changing the physical regions of the existing congested communication path or switching of the currently used spectrum band [10]. In both cases, the QoS requirements for the current SG communication transmission will be affected. Hence, the choice of switching activities should be made with respect to the requirements of different SG applications [15]. However, spectrum mobility passes interference to the current communication transmission. Because of this, schemes to prevent buffer overflows and minimize communication contention delay should be developed in order to allow for seamless, reliable and real-time monitoring in a CRSN based SG [21].

\subsubsection{Unique characteristics of CRSN}

CRSNs have numerous unique characteristics that differentiate them from the conventional wireless networks such as cellular/LTE, satellite/microwave and Wi-Fi. Since they incorporate the cognitive capabilities of CRN into a WSN they therefore differentiate themselves from CRN and WSN. Hence, a CRSN has unique features (possessing dualized features: CRN and WSN). These unique characteristics of a CRSN include:

- Capability of sensing the current radio frequency spectrum channel environment.

- Policy with configuration repository. Policies specify how the radio is to be operated, while the repository is formed usually from sources used to constrain the operating process of the radio so that it remains within regulatory or physical limits.

- Dynamic Spectrum Access (DSA) capabilities with multiple channels availability.

- Spectrum handoff capabilities

- Adaptive algorithmic mechanism. During the radio process, the cognitive radio is sensing its environment. It is following the constraints of the policy and configuration by exchanging with sensor nodes to best employ the radio spectrum and meet user demands.

- Low traffic flow.

- Reconfigurability and distributed cooperation capabilities.

- Limited memory and power constraints.

Due to the presence of these unique CRSN features, radio RRA schemes that are used for conventional wireless networks cannot be directly applied to a CRSN due to the dynamic availability of multiple channels in the CRSN and the dynamic spectrum access in the presence of primary user activity. Hence, 
CRSN Protocols for SGs applications

Spectrum sensing in CRSNs for

SG

Spectrum mobility in CRSNs for SG

Key immunity compliance requirements of communication infrastructure in SGs.

Table 2. Criteria for CRSNs deployment for SG applications compared with other applications

\begin{tabular}{cc}
\hline \hline $\begin{array}{c}\text { Unique criteria/constraints to be } \\
\text { onsidered in CRSNs deployment }\end{array}$ CRSNs for SG applications & $\begin{array}{c}\text { CRSNs for other } \\
\text { applications }\end{array}$ \\
\hline
\end{tabular}
spectrum sensing in CRSNs for SG in CRSNs for SG [21] [15]

Improved spectrum mobility scheme is required to prevent buffer overflows and minimize communication contention delay

Key immunity

compliance requirement is optional systems for SG utility automation [7]

CRSN Protocols for SGs includes CRB-MAC, CSMA/CA MAC, Distributed control algorithm (DCA), and RPL (routing protocol for low power and lossy networks) [8] - [11]

An optimized DSA is required for Generic DSA is used for spectrum sensing in CRSNs for other applications. Generic scheme is used for spectrum mobility in CRSNs for other applications.

\section{Related Works}

The RRA has been well investigated for various wireless networks, though not in the perspective of a SG. Numerous studies on RRA for different wireless networks such as cognitive radio networks (CRN), CRSN, and WSN, can be found in the literature [22]-[29]. These works are not in the context of a SG; they do not involve the integration of a wireless network into a SG in their surveys. Only a very few articles survey RRA from a CRSN perspective. Yet, their emphasis is not on the intersection of a CRSN in a SG for the RRA. This paper presents a survey that focuses on RRA in a CRSN based smart grid.

Surveys on RRA in a CRSN for SG environments have rarely been investigated. Refs. [30]-[32] survey works on RRA in terms of CR functionalities in a CRN. Ref. [30] conducted a survey on RRA in a WSN. Ref. [33] surveys works on RRA in a CRSN. In this work, CRSN resource allocation schemes are categorized and some optimization criteria highlighted for a CRSN. The work is not in the context of a SG. Other works which are not mainly concerned with the survey of resource allocation, but highlight some aspects of resource allocation strategies, are found in [34]-[42]. Ref. [34] presents a survey on 
strategy. Ref. [35] shows that resources in cognitive radio networks (CRNs) should dynamically be allocated according to the sensed radio environment.

Le and Hossain, in [36], presented a resource allocation framework specifically for spectrum underlay in cognitive wireless networks. Ref. [37] studies resource allocation in an Orthogonal Frequency Division Multiplexing (OFDM)-based cognitive radio network (CRN). This was with the consideration of many practical limitations such as imperfect spectrum sensing, limited transmission power, and different traffic demands of secondary users.

Table 3 presents a comparison of RRA surveys in CRN, CRSN and CRSN based SGs. It helps to show whether a survey of radio RRA has been considered in a CRSN based SG.

Table 3. Comparison table on radio resource allocation in CRSN based SG.

\begin{tabular}{lccccc}
\hline $\begin{array}{c}\text { Survey References } \\
\text { for resource } \\
\text { allocation }\end{array}$ & CRN & CRSN & $\begin{array}{c}\text { CRN } \\
\text { based SG }\end{array}$ & $\begin{array}{c}\text { CRSN } \\
\text { based SG }\end{array}$ & $\begin{array}{c}\text { Resource } \\
\text { allocation }\end{array}$ \\
\hline Tragos et al. [23] & Yes & No & No & No & Yes \\
Naeem et al. [24] & Yes & No & No & No & Yes \\
Ahmad et al. [33] & No & Yes & No & No & Yes \\
Ireyuwa et al. [34] & Yes & No & No & No & No \\
Xie et al. [35] & Yes & No & No & No & No \\
Le et al. [36] & Yes & No & No & No & No \\
Li et al. [37] & Yes & No & No & No & No \\
Yu et al. [38] & Yes & No & No & No & No \\
Khan et al. [57] & No & No & Yes & No & No \\
Akan et al. [43] & No & Yes & No & No & No \\
Gungor et al. [42] & No & No & Yes & No & No \\
Faheem et al. [59] & No & No & No & Yes & No \\
\hline \hline
\end{tabular}

Ref. [38] studied the energy efficiency aspect of spectrum sharing including power allocation in heterogeneous cognitive radio networks with femtocells. Ref. [39] proposed a correlation-based admission control strategy for efficient resource utilization in CRN. [40] proposed a distributed lightweight protocol for reduction of energy and communication overhead in CRSN. [31] presents throughput maximization for machine to machine communication using electromagnetic energy harvesting-based CRSN. [42] carried out investigative studies on WSN for SG.

Resource allocation was generally discussed in the above works, but the survey of resource allocation strategies was not their major target. Ref. [43] discussed issues regarding dynamic spectrum management in a CRSN. This work does not provide any survey on resource allocation strategies. Refs. [44] and [45] discuss CRN but RRA was not their main objective.

The authors in [46]-[50] carried out experimental work in RRA for CRN. Their experimental results validate improvements in some optimization criteria for resource allocation in a CRN. However, these works are not carried out from the perspective of a CRSN based SG.

Ref. [51] reported on experimental work in a CRN for the improvement of spectrum and energy efficiency using RF energy harvesting as an alternative data transmission for the SUs if the channel is occupied. However, the work does not involve RRA in a CRSN based SG nor evaluation of frequency spectrum for throughput improvement in a CRSN based SG. Ref. [52] carried out experimental work for RRA based on a CRN for a IoT sensor network. Though the work did not address a CRSN based 
SG. Ref. [53] proposed channel selection strategies in a CRN with Energy Harvesting for Internet of Everything.

449 Ref. [54] proposed a spectrum and energy harvesting enabled Heterogeneous Cognitive Radio Sensor

450

451

452

453

454

455

456

457

458

459

460

461

462

463

464

465

466

467

468

469

470

471

472

473

474

475

476

477

478

479

480
Network (HCRSN) for a RRA solution based on two algorithms that allocate the transmission time, power, fairness, and channels access including minimal energy consumption of the data sensors.

Ref. [55] conducted an experiment for RRA in a CRSN. The experimental results validate improved spectrum allocation, priority among sensor data, energy efficiency and reduce spectrum handoff. Experimental work was presented in [56] in which proposed energy efficient opportunistic spectrum allocation in a CRSN. However, this work does not involve RRA in a CRSN based SG.

Surveys of SGs that highlight some aspects of resource allocation are found in [12], [57] - [60]. They discuss spectrum sensing, they did not highlight resource allocation extensively such as including spectrum, QoS, fairness, priority, and power allocation schemes, etc. Resource allocation schemes were not their main focus. They did not consider evaluation of the frequency spectrum for throughput improvement in a CRSN based SG.

From the above discussion, some of the works focus on RRA in only CRNs or CRSNs, or other aspects of wireless networks without addressing the SG. None have surveyed the integration of resource allocation in a CRSN into a SG. The survey that involve a SG domain discussed some aspects of resource allocation without delving into the full details of the resource allocation scheme; and RRA is not the main aim of the articles.

Hence, this paper extends the work on RRA into the SG domain, as well as performance analysis of the frequency spectrum for throughput improvement in a CRSN based SG. Based on the literature, improvement of the throughput in a CRSN in a SG has rarely been investigated. Thus, the performance analysis work put forward here serves as the contribution to RRA in terms of the improvement of throughput. This contributes to other optimization criteria in a CRSN based SG.

\section{Radio Resource Allocation in CRSN Based SG}

\subsection{Radio resource performance improvement criteria}

RRA involves strategies or schemes of allocating radio resources such as frequency bands, transceiver power, time slots, handoff criteria, user fair allocation, modulation schemes, transmit antennas and sensing signal/channel detection probability, to the channel state information based on some performance improvement strategies or optimization criteria. Optimizing these radio resource criteria will go a long way to improve the overall performance of the CRSN in a SG environment. Hence, the aim is to utilize the limited spectrum, power constraints and network infrastructure efficiently. The following optimization criteria metrics are considered:

\subsubsection{Energy efficiency metric}

Realizing energy efficiency with power algorithm schemes is usually required to extend the lifetime of the battery of the sensor node. The energy efficiency criterion is necessary for a CRSN in a SG because the sensor nodes have limited power battery constraints. However, the schemes used for this criterion are based on energy preservation and power consumption minimization which cannot achieve maximum power performance. Energy/power efficient schemes for CRSN related applications in general and in the SG in particular have been widely studied [61]-[80]. Since SG applications are mission critical, it is essential to incorporate an energy harvesting scheme in the energy efficiency metric to provide a perpetual life for the sensor node. 


\subsubsection{QoS guarantee metric}

SGs have various applications with different and stringent QoS requirements. Hence, the resource allocation scheme design should consider different QoS support for a SG application. Resource allocation schemes involving CRSN applications that consider the QoS requirements are found in [69], [9], [10], [81]-[87]. Ref. [9] considered the QoS guarantee for heterogeneous traffic in a SG application such that each traffic type has an associated priority with specific QoS support. QoS support is imperative especially for SG surveillance and multimedia applications including distribution automation [88].

\subsubsection{Maximizing throughput metric}

Giving scheduling priority to data flows in terms of consumed network resources per amount of information transferred will help to maximize the total throughput of a CRSN based SG. Schemes utilizing throughput maximization scheduling based criterion in CRSN applications have been investigated in [70], [73], [74], [11], [89]-[95].

\subsubsection{Interference mitigation and avoidance}

Destructive interference from the external network to the CRSN based SG network should be avoided. Also, co-channel interference within the network as well as interference to the primary networks should be mitigated or cancelled. This interference avoidance and minimization criterion improves both the primary and secondary network. Resource allocation schemes that utilize this criterion in protecting the links of both the primary users and the secondary network have been studied in [11], [80], [94].

\subsubsection{Fairness scheduling criterion}

Fairness among SUs in opportunistic spectrum access and scheduling and fairness in transmission power allocation to SUs are essential in the design of RRA schemes for CRSN based SGs. Since there is trade-off between QoS guaranteed and maximum throughput and fairness, consideration of fairness between multiple sensor nodes when prioritizing traffic should be done in such a way that throughput improvement and QoS support are maintained. Work that utilized this fairness criteria in a SG is reported in [61]. They considered QoS guaranteed for heterogeneous traffic in SG applications such that each traffic type has an associated fairness. Resource allocation strategies that utilize fairness criteria are also found in [66], [11], [96]-[101].

\subsubsection{Priority scheduling criterion metric}

The need to prioritize various SG application traffic is essential so that it has the capability to adapt to varying network conditions in real time [102]. A typical traffic type are the control commands having small packet size [102]. Hence, prioritizing traffic types per their order of importance, bandwidth/spectrum demand, real time, and power of consumption is highly beneficial in the CRSN based SG domain. Prioritizing traffic in a CRSN based SG was also considered in [9], [100].

\subsubsection{Reduced Adaptive modulation overhead and probability of detection}

The adaptive modulation scheme in a CRSN based SG can dynamically adapt to other modulation types due to the DSA capability. This leads to an overhead as well as supplemental energy consumption that results in the event of adapting or switching to another modulation type [51] at the sensor node. Hence, there is need to design a resource allocation scheme in a CRSN based SG that has reduced complexity in terms of the adaptive modulation mechanism.

\subsubsection{Reduced spectrum handoff}


Spectrum handoff occurs too often in CRSN applications. This leads to overhead as well as extra energy consumption at the sensor nodes. When occurring during the hand-off, the buffer overflows result in packet losses and affects the transmission reliability. Works that make use of this criterion for the resource allocation in CRSN applications have been reported in [61], [63], [66], [71]. The authors in [64] presented a reduced handoffs technique using a home gateway (HGW) for a home area network in a cognitive radio-based SG. Ref. [47] investigated a resource allocation scheme involving reduced spectrum handoff for CRSN applications. Ref. [66] presented a dynamic spectrum access scheme that accomplishes the reduction in the number of spectrum handoff. The resource allocation algorithm in [47] also minimizes the spectrum handoffs.

The summary of the literature with respect to various resource optimization criteria used in different CRSN contexts has been tabulated in Table 4 . This table highlights each resource optimization criterion used in the different CRSN context including CRSN based SGs. It can be deduced from the table that the utilization of the optimization criteria for RRA in a CRSN based SG is limited. In this scenario, resource optimization criteria such as energy efficiency, throughput maximization, and adaptive modulation, are yet to be applied in a CRSN based SG. Hence, attention should be drawn to this.

Table 4. Summary of resource optimization criteria for CRSN based SG.

\begin{tabular}{lcccl}
\hline \hline $\begin{array}{c}\text { Resource } \\
\text { optimization } \\
\text { criterion }\end{array}$ & CRSN & $\begin{array}{c}\text { CRN } \\
\text { based SG }\end{array}$ & $\begin{array}{c}\text { CRSN } \\
\text { based SG }\end{array}$ & References for various optimization \\
\hline Energy efficiency & Yes & Yes & No & {$[63][64][61][66][70][74][104][107][20]$} \\
\hline QoS guarantee & No & Yes & Yes & {$[62][70][80]-[83][93][83][67][68]$} \\
\hline Throughput & Yes & No & No & {$[66][69][72][73][74][80][81][93]$} \\
\hline $\begin{array}{l}\text { Interference } \\
\text { mitigation }\end{array}$ & Yes & Yes & Yes & {$[63][66][69][75][81][90][109][110][19]$} \\
\hline Fairness & Yes & No & Yes & {$[63][66][81]$} \\
\hline Priority scheduling & Yes & Yes & Yes & {$[11][91][102]$} \\
\hline Adaptive modulation & Yes & No & No & {$[67][115]$} \\
\hline spectrum handoff & Yes & Yes & Yes & {$[62][63][108][71]$} \\
\hline \hline
\end{tabular}

\subsection{Radio resource allocation scheme architecture}

The RRA architectural strategy in a CRSN based SG is divided into four groups: centralized architecture, cluster architecture, distributed architecture and distributed heterogeneous architecture. The resource optimization criteria which have been highlighted in the preceding section are implemented using each specific resource allocation scheme architecture. These architectures will be looked at in turn.

\subsubsection{Centralized Architecture}

A centralized RRA scheme consists of the central node or sink node which serves as a base station that is responsible for providing network operation services such as spectrum allocation, power/energy control, node localization, link/modulation adaptation and routing among the sensor nodes. A logical topology for this architectural approach is a star network as illustrated in Figure 2. 
The centralized scheme can be classified in terms of how the information is processed, which includes the following: single sink, multi sink (for large coverage area and redundancy), and multiple task devices (for auxiliary devises and specific task within the network). RRA is made based on selected optimization criteria by the sink node which is then communicated to the sensor node. The selected optimization criteria may address more than one or two criteria. Centralized architecture schemes in CRSN related applications have been investigated in [61], [63]-[64], [65]-[68], [73], [11], [103]-[107], [89]-[91], [92] There are several advantages to centralized schemes. The main advantages include: (i) simplified energy efficiency management; and (ii) conflict avoidance in the transmission and reception link because the sink node coordinates every sensor node. However, there are some disadvantages in this architecture. The main disadvantages of these schemes include: (i) the network cannot support large density sensor nodes; and (ii) there is high signaling overhead leading to high energy consumption.

Notable RRA schemes that utilize centralized architecture are:

- Energy efficient joint source and channel sensing: A joint source and channel sensing scheme and power consumption minimization in a CRSN was proposed in [65]. The basis of this scheme is the perception of energy efficient joint source and spectrum sensing. The work involves two critical energy consuming tasks in a CRSN which are jointly considered. Specific and joint power consumptions are mathematically modeled to minimize the power consumption of each senor node.

- A home area network gateway (HGW) assisted cross-layer cognitive spectrum sharing mechanism was proposed in [61]. This was for a home area network (HAN) solution. The mechanism has two main algorithms: the spectrum access controller and power coordinator. These operate at the medium access control (MAC) and physical (PHY) layers, respectively. Each wireless sensor node in a HAN accesses the spectrum only if it is permitted by the centralized access controller. However, the power coordinator works in a decentralized architecture; it makes use of a noncooperative game between the wireless sensor nodes to adjust their transmitting power.

- Fair and energy efficient dynamic spectrum allocation: this scheme involves was presented in [66]. This scheme is for a low density CRSN. The sensor nodes are presumed to be located within a cell or segment boundary. The main objective of this scheme is to reduce handoff as well as signaling overhead. This is achieved by increasing the energy efficiency of an "interleaved FDMA" based CRSN and ensuring fairness between the spectrum sensor nodes. In this scheme, interference avoidance in the primary network is considered. This includes priority and fair spectrum allocation in the sensor nodes, and reflects the priority in the sensor data. Only the sensor nodes 
having data to transmit are assumed to send a spectrum resource request to the central nodes. Hence, this scheme supports unified multiple criteria goals.

- A hybrid dynamic spectrum access (H-DSA) strategy was proposed in [61]. This can significantly enhance the flexibility of communications infrastructure and spectrum efficiency, and improve a neighborhood area network (NAN). In this scenario, the spectrum bands in a NAN contain leased and licensed spectra from the telecommunication operator, which is referred to as the primary network, and the unlicensed spectra are used in an opportunistic manner.

- Energy Efficient Adaptive Modulation: a joint life-time maximization and adaptive modulation framework for realizing high power efficiency in CRSNs was presented in [67]. Adaptive modulation helps to improve the energy efficiency in a wireless network. This work considered a CRSN that contains uniformly distributed nodes within a low density area. This scheme performs adaptive modulation by utilizing parameters like time slot, synchronization, spectrum sensing, and Rayleigh fading characteristics. This scheme has the capability for interference detection and avoidance of the primary network.

- Energy Efficient Power Allocation: [73] investigated an energy efficient power allocation scheme for a CRSN. The aim of this scheme is to maximize the ratio of throughput to power. This work considers a CRSN such that each of the sensor nodes communicates on an orthogonal or at a right angle channel to the cognitive radio sink node or base station. There is a limit to the transmitted power of the sensor node. This is in order to limit the interference which is caused in the primary network to below a certain threshold.

- Cross-Layer Design for QoS Support: a cross-layer design that ensures the QoS requirement for CRSN based SGs was proposed in [9]. The varying characteristics of the data traffic for various applications in a SG means that the different QoS requirements need to handle the SG application traffic. This work handles the issues of heterogeneous traffic in a CRSN based SG by defining different classes of traffic with different priority levels. This classification is significant for separating the traffic with respect to the services and their network requirements e.g., latency, link reliability, and data rate.

- A hybrid guard channel (HGC) strategy has been proposed for cognitive NANs in a cognitive radio network based SG. The centralized scheme in [61] was designed with a hybrid guard channel that addresses the QoS of the sensor nodes and maintains it at a satisfactory level. This is because the dynamic nature of spectrum availability causes difficulty in stable and guaranteed QoS provisioning. The HGC strategy reduces overhead in the spectrum handoffs, this is achieved by reserving a certain number of channels in both the licensed and unlicensed bands for the use of spectrum handoffs.

\subsubsection{Cluster Architecture}

On a topology level, cluster architecture is obtained by grouping the CRSN nodes within a smaller subnetwork transmission area. A designated node usually known as Cluster Head $(\mathrm{CH})$ controls this group of sensor nodes as shown in Fig. 3. The $\mathrm{CH}$ performs a similar role of allocating resources as the sink node in a centralized scheme. However, the $\mathrm{CH}$ has less overhead and utilizes less power for the common control channel in each cluster compared to the sink node in a centralized scheme. Hence, this scheme can achieve better spectrum use with the help of the distribution of nodes in several clusters, and with bandwidth reuse. Cluster schemes have been studied in [94], [92], [82], [75], [92], [84]-[85], [108]-[110]. A close cluster member can perform the role of the $\mathrm{CH}$ if the $\mathrm{CH}$ fails. Since there is a small number of cluster members in each cluster, this leads to low signaling overhead at each $\mathrm{CH}$ compared to the overhead at the sink node of a centralized architecture. The main advantages of this scheme are: (i) information is local since a sensor node keeps the information of its neighboring node within a cluster; (ii) the cluster architecture is scalable; and (iii) reconfiguration is done locally on only the affected part. 
However, there are some drawbacks with this architecture. The main drawback is the high number of broadcasts which is equal to the number of clusters; thus, leading to a broadcast storm in the network.

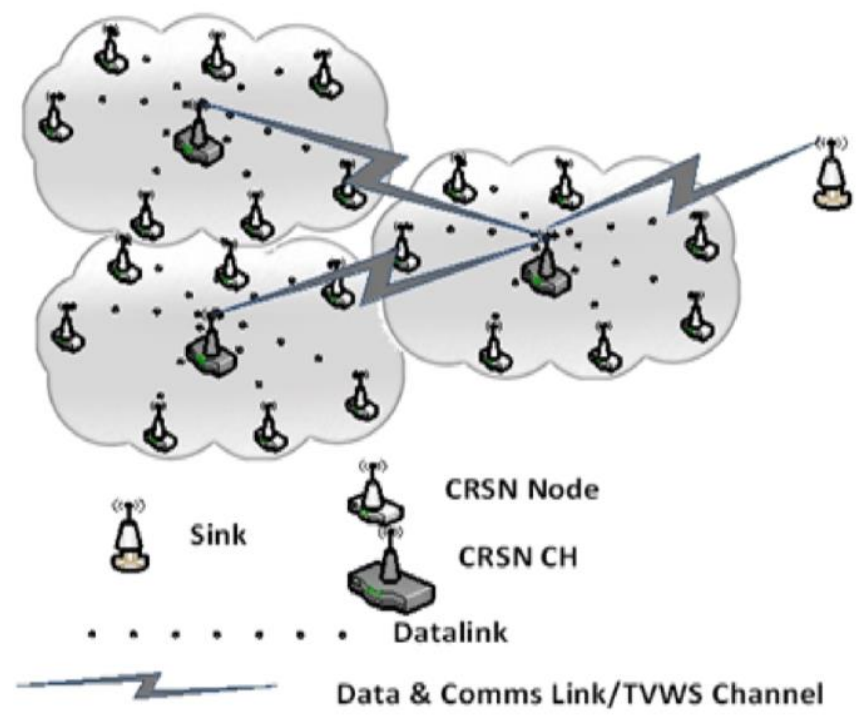

Figure 3. Cluster resource allocation architecture for a CRSN based SG

Notable RRA schemes that utilized the cluster architecture are:

- Periodical sensing (PS) scheme: this scheme was proposed for a WiMAX based CR system network to manage co-channel band interferences during usual communication in power distribution substation monitoring. Ref. [94] grouped the PS data into time and frequency domains such that the interference is classified into various types. It then uses this classification to execute a corresponding management method in order to minimize the interference. This will help to avoid the in-band interference that results from other communication devices operating at the same frequency with the SCADA in the SG environment.

- Energy efficient channel management: a cluster-based energy efficient channel management framework for CRSN applications has been proposed in [75]. This scheme is based on partially observable Markov decision process framework. The work involves a small network connected in star topology and with a $\mathrm{CH}$ and multiple cluster members. channel sensing and channel switching are considered in this work. The scheme manages energy efficiently by making the CRSN to operate on a channel tagged operating channel that is not occupied by the primary network while maintaining another vacant channel. as a backup.

- Joint node selection and channel Allocation: in [78], a scheme that selects the optimal number of sensor nodes with an efficient channel allocation mechanism was proposed. This scheme improves the performance of a cluster architecture based CRSN. In this work, clustering is achieved using the K-means clustering mechanism [111]. The problem of node selection is formulated as a knapsack problem, whereby a $\mathrm{CH}$ in each cluster controls the optimal number of sensors and selects the suitable sensors. After which, the Hungarian algorithm [112] is used for efficient channel allocation between the sensors thereby prolonging the network lifetime and giving appreciable data transmission in the sensor nodes.

- Energy efficient spectrum sensing: in [98], an energy efficient spectrum sensing node selection for cooperative channel sensing was proposed. The scheme involves energy conservation and precise spectrum sensing under a network of limited energy availability. In this scheme, the sensor nodes liaise and form coalitions for collaborative sensing. In each coalition or cluster, one sensor node is chosen as the cluster head which makes sensing decisions in a centralized manner at the cluster 
level. Between the sensor nodes of each coalition, the cluster head selects only the most suitable nodes for cooperative sensing.

- Markov chain modeling of a CRSN in SG: this scheme was presented in [109]. It aims at reducing transition delay during handoffs. The authors use examples of Markov chain models. The primary networks have prioritized access to the spectrum compared to the CRSN users, and are unaware of the CRSN user usage of the spectrum. Thus, the primary user arrivals follow a Poisson distribution with rate $\lambda_{p}$, and their service time is exponentially distributed with rate $\mu_{p}$. Likewise, CRSN secondary users follow a Poisson distribution with rate $\lambda_{s}$ and exponential service rate $\mu_{s}$. A CRSN user is forced to immediately relinquish a channel due to the arrival of any primary network and instantaneously transition into other available spectrum resources.

- Energy efficient spectrum aware clustering: in a cluster architecture CRSN, the selection of a suitable $\mathrm{CH}$ together with the determination of an optimal number of clusters are essential in energy and spectrum efficiency. In [12], an energy efficient clustering scheme is considered. This work is centered on finding the optimal number of clusters to reduce transmission power consumption and on avoiding interference to the primary network. In this work, two types of communication are considered: intra-cluster and inter-cluster communication. In intra-cluster communication, the sensor nodes transmit their collected information to the matching $\mathrm{CH}$, whereas in inter-cluster communication, the $\mathrm{CH}$ compresses the aggregated collected data and sends it to the neighboring relaying $\mathrm{CH}$ for subsequent transmission to the sink node.

\subsubsection{Distributed architecture}

In a distributed architecture scheme, each CRSN node makes its transmission decision in an independent manner. In addition, neighboring sensor nodes can cooperate with each other for transmission decisions. There is no central or base station node among the sensor nodes to coordinate the communication. Distributed resource allocation schemes can either have a cooperative distributed resource allocation or non-cooperative distributed resource allocation.

These schemes can quickly adjust to changes, and are robust to time changing wireless environments. For example, if an area of the network is disturbed, only the sensor nodes in the affected area will need to update their transmission mechanism which is a relatively faster process; whereas in the case of a centralized architecture, the resource allocation for all the sensor nodes will be updated.

In addition, the distributed schemes have lower signaling overhead as well as a faster decision process. The advantages of distributed schemes are similar to cluster schemes; however, with an additional advantage of reduced energy consumption at every sensor node.

The major disadvantage is that connectivity cannot be assured since each node makes decisions on local information which may include error or malicious activity spread by the neighboring nodes which renders distributed resource allocation to a weak optimal solution. Distributed architecture resource allocation in CRSN related applications has been studied in [62], [108], [80], [85]-[86], [88], [93], [113]. An example of a distributed resource allocation architecture for a CRSN based SG is shown in Figure 4. 


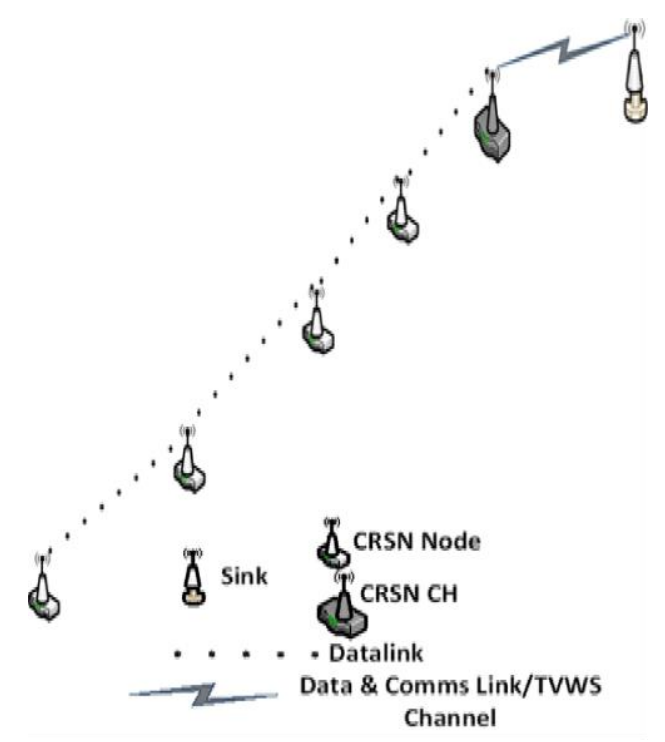

Figure 4. Distributed resource allocation architecture for CRSN SG.

711

Notable RRA schemes that utilize a distributed architecture are:

- Spectrum discovery schemes were presented in [62]. The schemes comprise of non-cooperative spectrum discovery and cooperative spectrum discovery. The objective of these schemes is to reduce the total energy of consumption of the sensor nodes during sensing using a home gateway (HGW). The schemes involve setting the threshold of the detection probability and the threshold of the false alarm probability, respectively. The thresholds represent the guarantee of sensing performance. Hence, an energy minimization problem in a scenario with two channels was formulated.

- Energy efficient spectrum access: distributed energy efficient power allocation and a sub-carrier selection framework for a multi-carrier CRSN was proposed in [80]. This distributed framework allocates power and a subcarrier to each $\mathrm{CR}$ sensor node based on the data rate requirement and power flow. This increases the energy efficiency of the network as well as avoiding any destructive interference to the primary network and the existing sensor nodes. Hence, it reduces the energy consumption of all the subcarriers allocated to the sensor nodes, thereby maximizing the network lifetime, and giving an appreciable QoS support.

- Robust distributed power control: a distributed power control algorithm was presented in [74]. The algorithm maximizes the throughput and energy efficiency of industrial CRSNs. In this work, the sensor nodes transmit data to the CRSN base station with the aim of maximizing the total rate of all the sensors at the base station. The scheme ensures that the SINR of each sensor is above a threshold such that the cumulative interference caused to each primary network by all the sensor node transmissions is brought below a predefined threshold.

- Energy efficient packet size optimization: in [87], a framework where each sensor node autonomously determines the optimal packet size before transmission was proposed. The main aims of this work are to minimize energy consumption, improve transmission efficiency, offer protection to a primary network, and increase event detection reliability. The energy efficiency of a CRSN can be enhanced by shaping the energy efficient packet size. Energy efficient packet size shaping is an active area of research for wireless networks.

- Channel packing scheme (CPS): a novel non-cooperative sensing scheme called a channel packing scheme (CPS) was proposed in [88]. This scheme integrates the role of optimal channel sensing into the analysis of the heterogeneous CRSN system performance to alleviate the problem encountered in serial search (SS) or random search (RS) sensing in heterogeneous CRSN based SG 
networks. That is, unnecessary secondary user blocking. CPS consists of two steps. The first step involves the incoming sensor node or user with less bandwidth requirement, which identifies a channel that includes sub channels already occupied by other sensor nodes or users of the same type. For the second step, the first available sub channel in sequence is allocated for this new sensor node or user. It is assumed that each channel is composed of $r$ sub channels.

- Spectrum-aware and cognitive sensor networks (SCSNs) were presented in [94]. These have a distributed scheme architecture. The schemes aim to overcome varying spectrum characteristics and severe environmental conditions for SG applications in a sensor network. The distributed spectrum-aware sensor nodes monitor critical SG equipment such that sensed data will be dynamically sent over available spectrum bands in a multi-hop manner to meet the applicationspecific requirements [113].

Table 5 summarizes the schemes with multiple optimization criteria consideration as well as cross layer framework consideration in different CRSN contexts. From the table, with respect to the references, it is obvious that many RRA schemes have been applied to CRSN applications in general whereas only very few are applied to CRN based SGs and CRSN based SGs. Schemes with multiple optimization criteria, that is, schemes having two or more resource optimization criteria, are very few with regards to CRSN based SGs. In addition, only one scheme with a cross layer framework is applied to a CRSN based SG. Utilizing a cross layer framework in RRA will improve communication in a SG. This is because the protocol stack in the bottom and upper layers of the sensor nodes and wireless device will exchange information seamlessly through a common control channel without delay and complexity. In general, a scheme with multiple optimization criteria and a cross layer framework will improve radio RRA in a CRSN based SG.

\subsubsection{Distributed Heterogeneous Clustered (DHC) Architecture}

The DHC architecture from a recent work [114] can be adopted for a CRSN based SG deployment in order to leverage multiple performance improvement criteria. The architecture consists of heterogeneous CRSN nodes such as normal ZigBee CR nodes, actuator, and multimedia sensor nodes. It is responsible for providing network operation services such as spectrum allocation, power/energy control, node localization, link/modulation adaptation and routing among the sensor nodes. A logical topology for this architectural approach is illustrated in Figure 5. The allocation of radio resources here is done in a distributed clustered manner covering an extensive and long range area. This scheme is suitable for a SG application, based on the fact that a SG requires heterogeneous networks in supporting different QoS for the various SG applications. Since this architecture is a newly introduced scheme, only very few schemes utilize this architecture for RRA in a CRSN based SG. The main importance of the DHC architecture is that it circumvents the disadvantages in centralized and distributed architecture while leveraging all the benefits of other architectures.

DHC architectures consider the EMC in order to operate optimally in a varying EMI SG environment. These schemes can quickly adjust to changes, and are robust to time varying wireless and EMI environments. Notable schemes are found in [38], [108], [115]. Ref. [38] proposed the energy efficiency aspect of spectrum sharing including power allocation in heterogeneous CRNs using a Stackelberg game with femtocells. Though this scheme is not specifically for the SG environment. Ref. [108] proposed a queuing theoretic model of the important components of a CRSN using the bandwidth of a heterogeneous network, including service rate heterogeneity and proactive priority for primary users. Ref. [115] proposed a probability of detection mechanism using a moment generating 
Table 5. Summary of cross-layer framework with respect to various RRA schemes for CRSN based SG.

\begin{tabular}{|c|c|c|c|c|c|}
\hline $\begin{array}{l}\text { References for } \\
\text { various resource } \\
\text { allocation schemes }\end{array}$ & CRSN & $\begin{array}{c}\text { CRN } \\
\text { based SG }\end{array}$ & $\begin{array}{c}\text { CRSN } \\
\text { based } \\
\text { SG }\end{array}$ & $\begin{array}{l}\text { Scheme with } \\
\text { multiple } \\
\text { optimization } \\
\text { criteria }\end{array}$ & $\begin{array}{c}\text { Cross layer } \\
\text { framework } \\
\text { consideration }\end{array}$ \\
\hline Yu et al. [61] & No & Yes & No & Yes & Yes \\
\hline Byun et al. [63] & Yes & No & No & Yes & No \\
\hline Zhaoyang et al. [65] & Yes & No & No & Yes & Yes \\
\hline Sun et al. [69] & Yes & No & No & Yes & No \\
\hline Gao et al. [70] & Yes & No & No & No & No \\
\hline Ayala et al. [71] & Yes & No & No & No & No \\
\hline Naeem et al. [76] & Yes & No & No & No & No \\
\hline Shah et al. [11] & No & No & Yes & Yes & Yes \\
\hline Khalil et al. [91] & No & No & Yes & No & No \\
\hline Lin et al. [96] & No & Yes & No & No & No \\
\hline Izumi et al. [107] & Yes & No & No & No & No \\
\hline Zhang et al. [65] & No & Yes & No & No & No \\
\hline Han et al. [78] & Yes & No & No & No & No \\
\hline Hareesh et al. [87] & Yes & No & No & No & No \\
\hline Liang et al. [85] & Yes & No & No & Yes & No \\
\hline Alagoz et al. [88] & Yes & No & No & Yes & No \\
\hline Seneviratne et al. & Yes & No & No & Yes & No \\
\hline$[89]$ & Yes & No & No & Yes & No \\
\hline Phuong et al. [96] & Yes & No & No & No & No \\
\hline Hu et al. [104] & No & No & Yes & Yes & No \\
\hline Luo et al. [111] & Yes & No & No & No & No \\
\hline Aslam et al. [80] & Yes & No & No & No & No \\
\hline Lee et al. [77] & Yes & No & No & No & No \\
\hline
\end{tabular}

786

787 function and a maximum ratio combiner (MRC) for performance improvement of RRA in a 788 multichannel CRSN based SG.

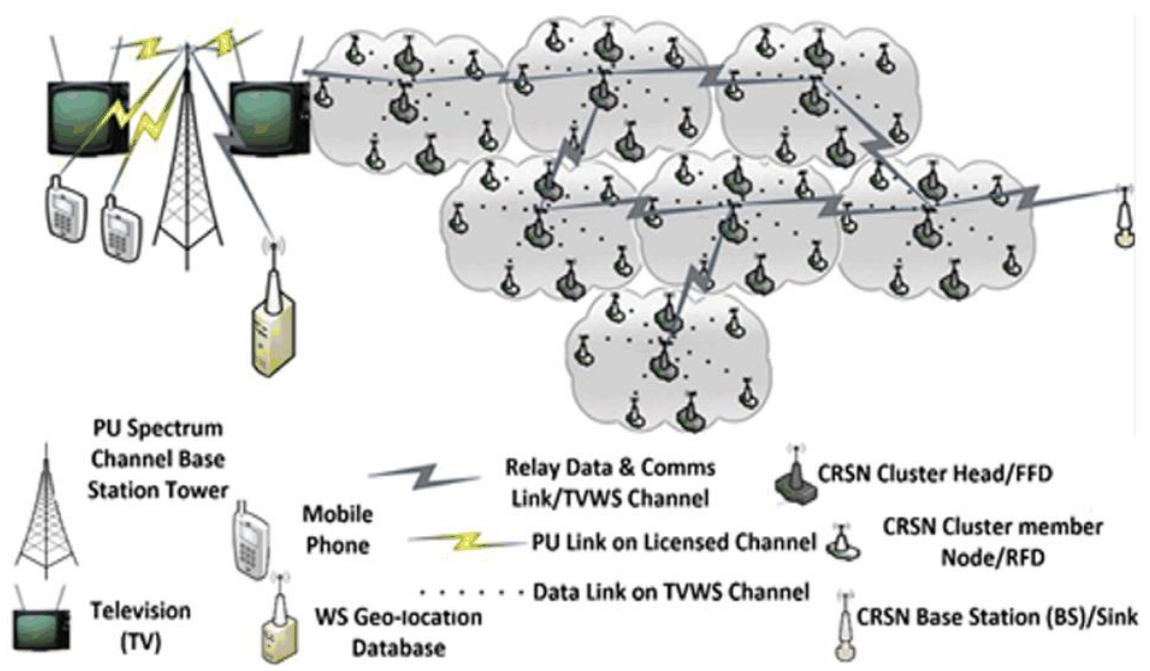




\section{Channel Allocation for Improved Throughput in CRSN based SG}

The available channels or spectrum holes are dynamically allocated by the SU base-stations to each SU for communication. However, high bit error probability or blocking probability in the SU network is a major problem associated with channel allocation in CRSNs for SG. This problem ultimately causes poor throughput. Hence, it is important to mitigate against the problem of blocking probability, in order to obtain maximised throughput of the channel allocation.

\subsection{Guaranteed Network Connectivity Channel Allocation for Throughput Maximization in CRSN-based $S G$}

The algorithm below, which involves guaranteed network connectivity channel allocation for throughput maximization (GNC-TM) algorithm has been introduced in a CRSNs for SG. An equilateral triangulation pattern graph is employed in the GNC-TM algorithm. The equilateral triangulation pattern graph is denoted as $G=(V, E)$, where $V$ represents the vertices of the triangle and $E$ the edges which is the communication links or line segments between the vertices.

The SU base-station or cluster Head $(\mathrm{CH})$ coordinates the opportunistic channel access from the PU networks via DSA. It allocates the readily available unused channels to the sensor nodes at the MAC protocol layer through the CSMA/CA. Up to six channels in the 650-860 MHz frequency band can be readily available when it is not used by the PUs. The SU or CRSN nodes automatically hand over the channels as soon as the PU arrives. This intermittent arrival and relinquishing of the channels can cause unnecessary delay or blocking probability to the CRSNs. To address this, a common backup channel (CBC) and GNC-TM algorithm can be introduced as shown in Algorithm 1. The CBC serves as the control channel and handles the control signaling of the SUs as the communication channel when the available channels are in use by the PUs. The GNC-TM algorithm commences with the six available channels (AC). The seventh channel is taken to be the $C B C$; and the vertices are represented as $a 1, b 1$, $c 1, a 2, b 2, c 2, \ldots \ldots . . a n, b n, c n$, which indicate connections with channels. Relating to Algorithm 1, lines 7 and 8 , the vertices can be connected by the available or $\mathrm{CBC}$ channel. Once connected, channels are then allocated to the associated sensor node for communication and exchange of messages or sensed data.

The allocated channel signals can be modulated with lower constellation order $M$, for $(M=4)$ of quadrature amplitude modulation (QAM) under Rayleigh fading channel distribution conditions. Hence, the average received signal-to-noise ratio (SNR) signal denoted as $\bar{\gamma}$ for each channel, can be expressed as

$$
\begin{gathered}
\bar{\gamma}=E_{S} / N_{0} \\
\bar{P}_{E}=a / n\left\{\frac{1}{b \bar{\gamma}+2}-\frac{a}{2} \times \frac{1}{b \bar{\gamma}+1}+(1-a) \sum_{i=1}^{n-1} \frac{S_{i}}{b \bar{\gamma}+S_{i}}+\sum_{i=1}^{2 n-1} \frac{S_{i}}{b \bar{\gamma}+S_{i}}\right\}
\end{gathered}
$$

where $E_{S}$ denotes the average transmission power or energy per symbol in the channel, and $N_{0}$ denotes the Gaussian noise power per bandwidth of a channel. To obtain an appreciable or higher received average SNR, the error or blocking probability should be minimal. But the error or blocking probability, $\bar{P}_{E}$ of the MQAM signal under Rayleigh fading channel is given by [114]: 


\section{Algorithm 1}

830

831

836

837

838

839

840

841

842

843

844

845

846

847

848

849

850

851

852

853

854

855

856

\section{GNC-TM: Guaranteed network connectivity channel} allocation for throughput maximization

\section{BEGIN}

1. $G=\{V, E\}$;

2. $A C=[1,2,3,4,5,6]$;

3. $C B C=[7]$;

4. $V=\{a 1, b 1, c 1, a 2, b 2, c 2, \ldots . . . a n, b n, c n\}$;

5. $\quad \mathrm{E}=\{\mathrm{a} 1, \mathrm{~b} 1 ; \mathrm{a} 1, \mathrm{c} 1 ; \mathrm{b} 1, \mathrm{c} 1 ; \mathrm{a} 2, \mathrm{~b} 2 ; \mathrm{a} 2, \mathrm{c2} ; \mathrm{b}, \mathrm{c} 2$; an,bn; an, cn; bn,cn\};

6. if $A C=1|| 2|| 3|| 4|| 5|| 6$;

7. ECONNECTED $=\mathrm{AC}(\mathrm{an}, \mathrm{bn} ; \mathrm{an}, \mathrm{cn} ; \mathrm{bn}, \mathrm{cn})$;

8. else if $A C=7$;

9. $E_{C B C}=C B C(a n, b n ; a n, c n ; b n, c n)$;

10. while $E=E$ CONNECTED;

11. Send msg via $A C$

13. else

14. send control signal and msg via $C B C$; end if;

15. end

16. End

where $a=1-\frac{1}{\sqrt{M}} ; b=\frac{3}{M-1} ; s i=2 \sin i \pi / 4 n ; M$ is the constellation order $(M=4)$; and $n$ is the number of iterations.

The relationship of SNR and throughput is given to obtain the maximized throughput so that

$$
\text { Throughput }=C B \times \log _{2}(1+S N R)
$$

where $C B$ is the channel bandwidth

\subsubsection{Simulation experimental setup for GNC-TM channel allocation}

In this section, the GNC-TM algorithm was implemented with error probability and signal throughput in the MATLAB environment. Table 6 shows the simulation parameters. The GNC-TM model is run and the results compared with existing protocol. The performance efficiency of the GNC-TM model is evaluated based the following metrics: error probability and throughput.

\subsubsection{Simulation Results and Analysis of GNC-TM channel allocation in CRSN for SG}

Figure 6 shows the throughput maximization analysis of the channel allocation based on bit error rate for the GNC-TM model compared with the existing Protocol. The results confirm that the GNC-TM model can effectively do throughput maximization in channel allocation with minimal error rate and high throughput. Figure 6 shows the GNC-TM minimal error probability starting with less than $10^{-2}$ and ending with $10^{-5}$. Existing protocol error probability starts st about $10^{-1}$ and ends at $10^{-4}$. 
Table 6. GNC-TM model simulation parameters

\begin{tabular}{ll}
\hline \hline Parameters & Value \\
Simulation runs (n) & 10,000 \\
Multi-path fading & Rayleigh fading \\
SNR & $0: 3: 24 \mathrm{~dB}$ \\
Modulation size & $4 \mathrm{QAM}$ \\
Channel Bandwidth (CB) & $6 \mathrm{MHz}$ \\
Shadow Fading & Log-Normal Shadowing \\
\hline
\end{tabular}

858

859 In a similarly manner, the maximum throughput in the GNC-TM is $85 \mathrm{Mbps}$, while that of the existing 860 protocol is $28 \mathrm{Mbps}$. Therefore, the results validate both the throughput maximization and error 861 reduction in the GNC-TM model where the throughput and error rate are improved compared to existing protocols for channel allocation in CRSN-based SGs.

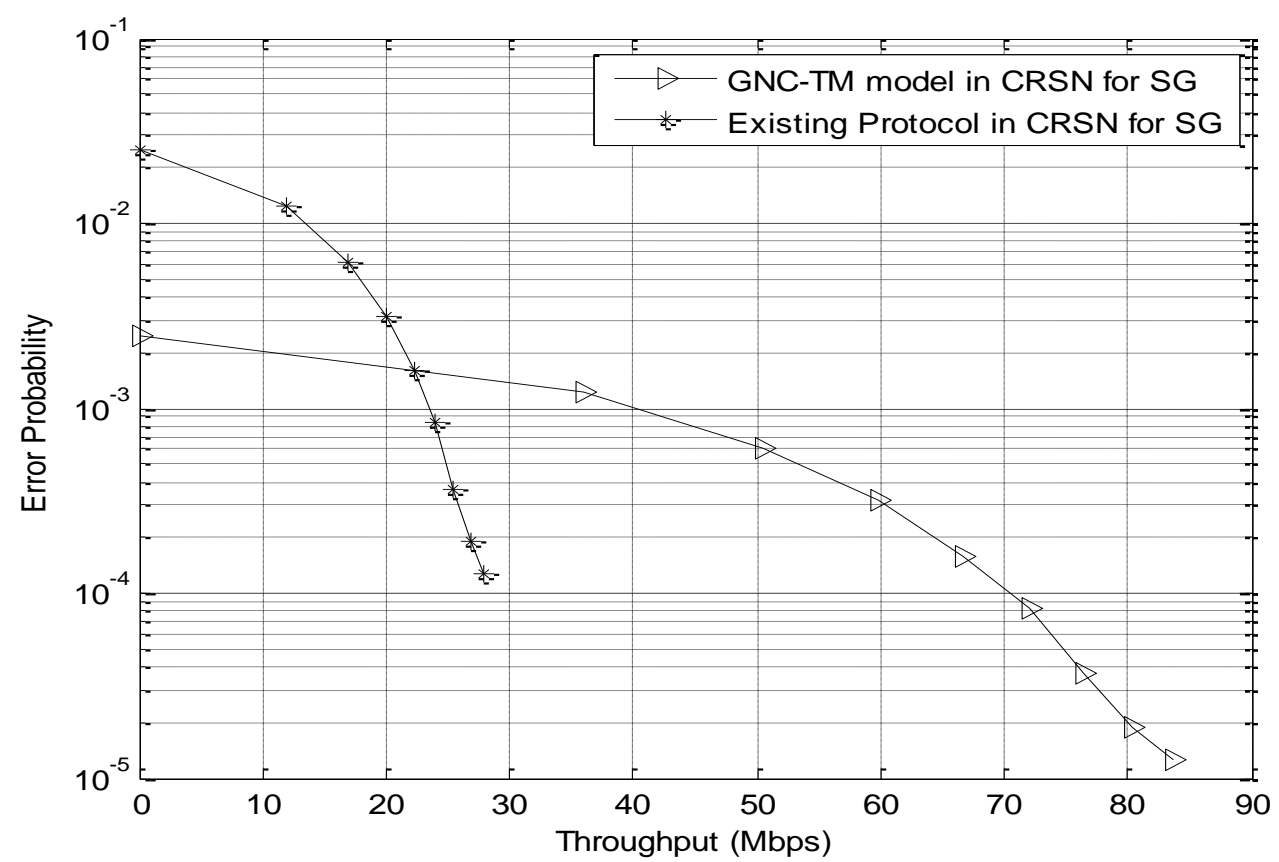

863

Figure 6. Throughput evaluation of channel allocation in CRSN for SG

\subsection{Optimal spectrum band determination in RRA for throughput improvement criteria in CRSN based SG}

4.2.1. Concepts and simulation experimental setup for optimal spectrum band determination

PU activities can impact on the performance of the SUs or CRSN users. Frequent PUs activities will lead to fewer spectrum holes. However, multiple SU spectrum channels will lead to more spectrum holes or white space. Multiple channels and high bandwidth is adequate for the enhancement of the throughput of the SUs [116]. Hence, making CRSN users operate at a higher frequency band (UHF: 470-868 MHz or higher) during certain PU activities will create more channels thus improving the throughput performance of the CRSN users. Whereas a lower frequency band (VHF: 54-216 MHz) for the CRSN 
users, operating with the same conditions as the PU activities, will adversely impact on the throughput performance of the CRSN users due to limited spectrum holes and fewer channels.

An investigation was carried out using NetSim simulation and modelling software for the performance analysis of the SU or CRSN throughput in order to establish a suitable spectrum band for the CRSNs in a SG network. NetSim is a network Discrete Event Simulation (DES) software package for protocol modelling and simulation. It allows for analyses of networks with unmatched depth [117]-[118].

Table 7 shows the network parameters used for modelling a CRSN base station and CRSN module users in three spectrum bands: $54-80 \mathrm{MHz} ; 54-216 \mathrm{MHz}$; and 54-802 MHz respectively. The experiment was modelled with a SG custom application. The SG application is generated from the SG application server with a packet size of 1460 bytes, which is then used by twenty CRSN modules for the SG data services. Table 8 shows the SG application parameters.

Table 7. CRSN configuration parameters

\begin{tabular}{|lc|}
\hline \multicolumn{2}{c|}{ CRSN Base station parameters } \\
\hline Device Name & Base Station \\
Min/Max Frequency & $54 / 862 \mathrm{MHz}$ \\
Coding rate & $(1 / 2)$ \\
Distance (Range) & $1 \mathrm{~km}$ \\
Channel Bandwidth & $6 \mathrm{MHz}$ \\
Modulation & $4 \mathrm{QAM}$ \\
Pathloss & $30 \mathrm{~dB}$ \\
Transmission Power & $5 \mathrm{~mW}$ \\
Frequency (varies with each & $54-80 \mathrm{MHz} / 54-216 \mathrm{MHz} / 54-802$ \\
scenario) & $\mathrm{MHz}$ \\
\hline \multicolumn{2}{c}{ CRSN Module parameters } \\
\hline Device Name CRSN Module \\
Transport Layer protocol & $\mathrm{UDP}$ \\
Pathloss & $30 \mathrm{~dB}$ \\
Transmitter power & $5 \mathrm{~mW}$ \\
\hline \hline
\end{tabular}

Table 8. SG Application parameter

\begin{tabular}{|ll|}
\hline \hline Device Name & SG Application Server \\
Application Method & Broadcast \\
Application Type & Custom \\
Application Name & DRM \\
Source ID & SG Application Server \\
Destination ID & CRSN Modules \\
Start Time (s) & $0 \mathrm{~s}$ \\
End time (s) & $100 \mathrm{~s}$ \\
Packet size (byte) & 1460 \\
\hline \hline
\end{tabular}

4.2.2 Simulation results and analysis of throughput based on spectrum band determination in CRSNs for SG

The simulation was conducted in Netsim under the same severe propagation conditions ( $30 \mathrm{~dB})$ of SG in three different spectrum bands: $54-80 \mathrm{MHz} ; 54-216 \mathrm{MHz}$; and 54-802 MHz respectively. The aim of 
the simulation experiment is to analyse the throughput of the CRSN link in different spectrum bands with severe SG environmental conditions. There are the same PU activities in the three scenarios in order to ascertain a suitable frequency spectrum for an optimal throughput. A data packet of 1460 bytes for the SG application was transmitted to be received by the CRSN nodes. The results of the CRSN link moving average throughput were obtained and are shown in Figures 7 to 9.

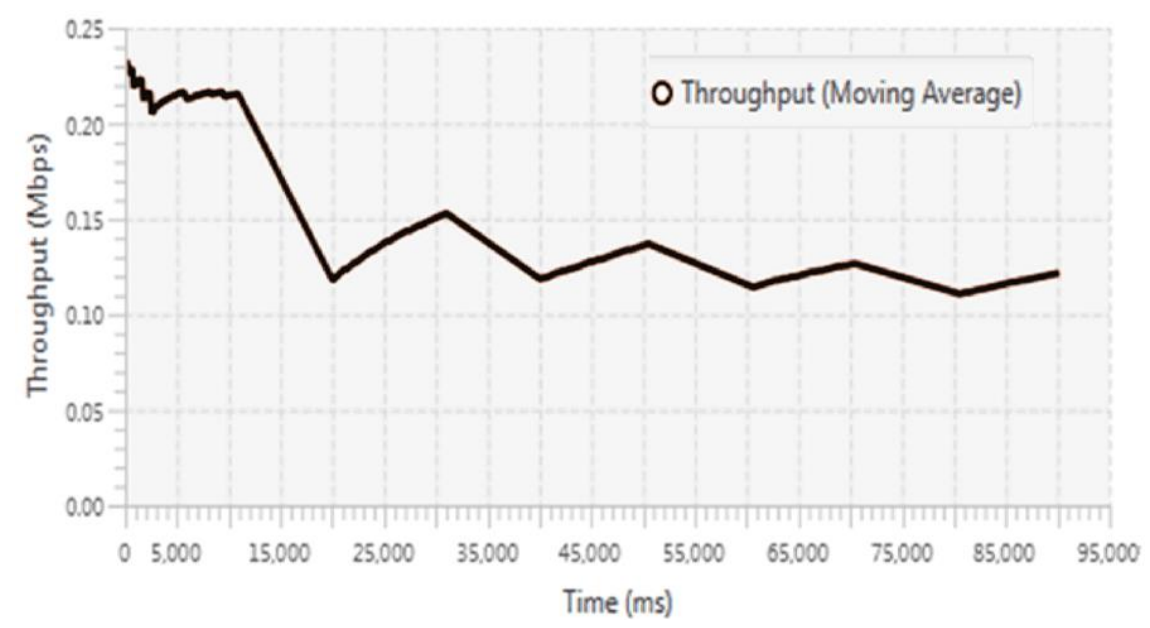

Figure 7. Scenario 1: $54 \mathrm{MHz}-88 \mathrm{MHz}$

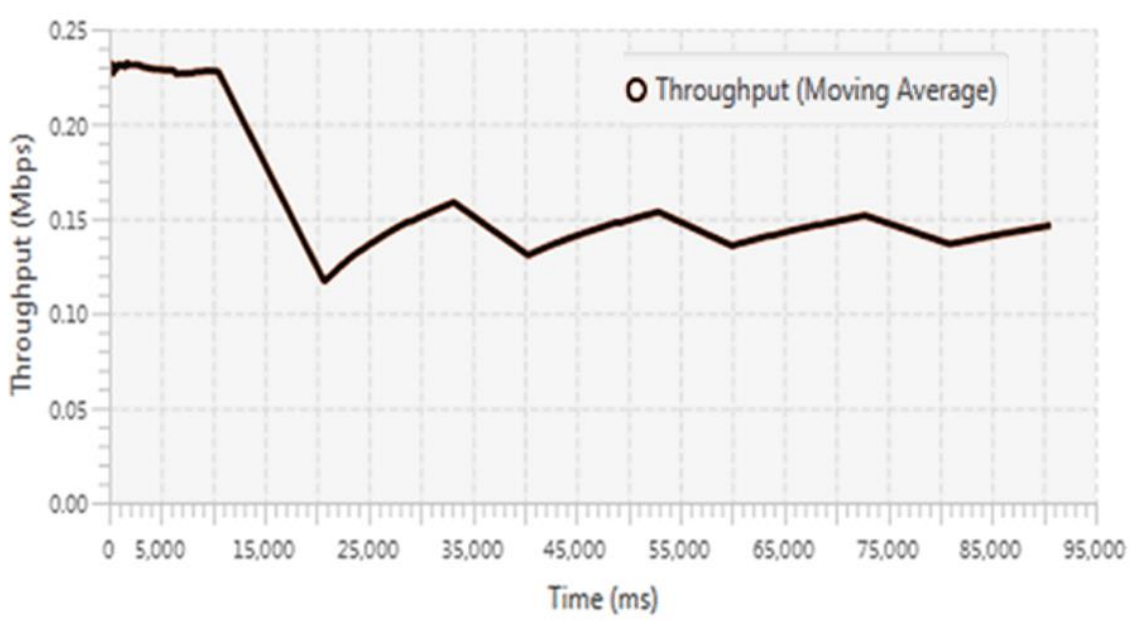

Figure 8. Scenario 2: $54 \mathrm{MHz}-216 \mathrm{MHz}$

Figure 7 shows Scenario 1. A moving average throughput of $0.23 \mathrm{Mbps}$ is obtained at the initial phase of the transmission. This reduces then levels off up to about $10000 \mathrm{~ms}$. It then reduces to $0.12 \mathrm{Mbps}$ at $17500 \mathrm{~ms}$. It increases again to about $0.15 \mathrm{Mbps}$ at $31000 \mathrm{~ms}$ and decreases. It then continues erratically with attainment of below $0.15 \mathrm{Mbps}$ throughput throughout the transmission duration.

Figure 8 shows Scenario 2. A moving average throughput of $0.23 \mathrm{Mbps}$ is initially obtained and this starts reducing at about $10000 \mathrm{~ms}$ and resumes at about $20000 \mathrm{~ms}$. A throughput of $0.15 \mathrm{Mbps}$ is attained at $30000 \mathrm{~ms}$. It then starts reducing again at $33000 \mathrm{~ms}$. It continues erratically with an attained throughput that is about $0.15 \mathrm{Mbps}$ throughout the transmission duration.

Figure 9 shows Scenario 3. A moving average throughput attainment of $0.23 \mathrm{Mbps}$ at the initial phase of the transmission. This continues steady with negligible throughput fluctuation, and maintains $0.23 \mathrm{bMbps}$ throughout the transmission duration. 
912 Overall, the higher frequency spectrum with more channel availability gives a steady throughput. This gives rise to optimal appreciable throughput of the CRSN in a SG. Because the throughput is necessary for network connectivity in the CRSNs radio resources such as a spectrum channel to be efficiently allocated to CRSN nodes. Whereas, the lower the frequency spectrum, which usually has less available channels, has lower throughput attainment with unsteady conditions. This latter case is not suitable for SG applications that are mission critical. The higher spectrum bands are associated with more channels compared with lower frequency bands which are usually associated with less available channels.

919

920

921

922

Hence, a CRSN for SG communications should be developed to accommodate higher spectrum bands with multiple available channels of over $800 \mathrm{MHz}$ bands in order to leverage spectrum hole from both digital TV and some 4G/LTE frequency bands.

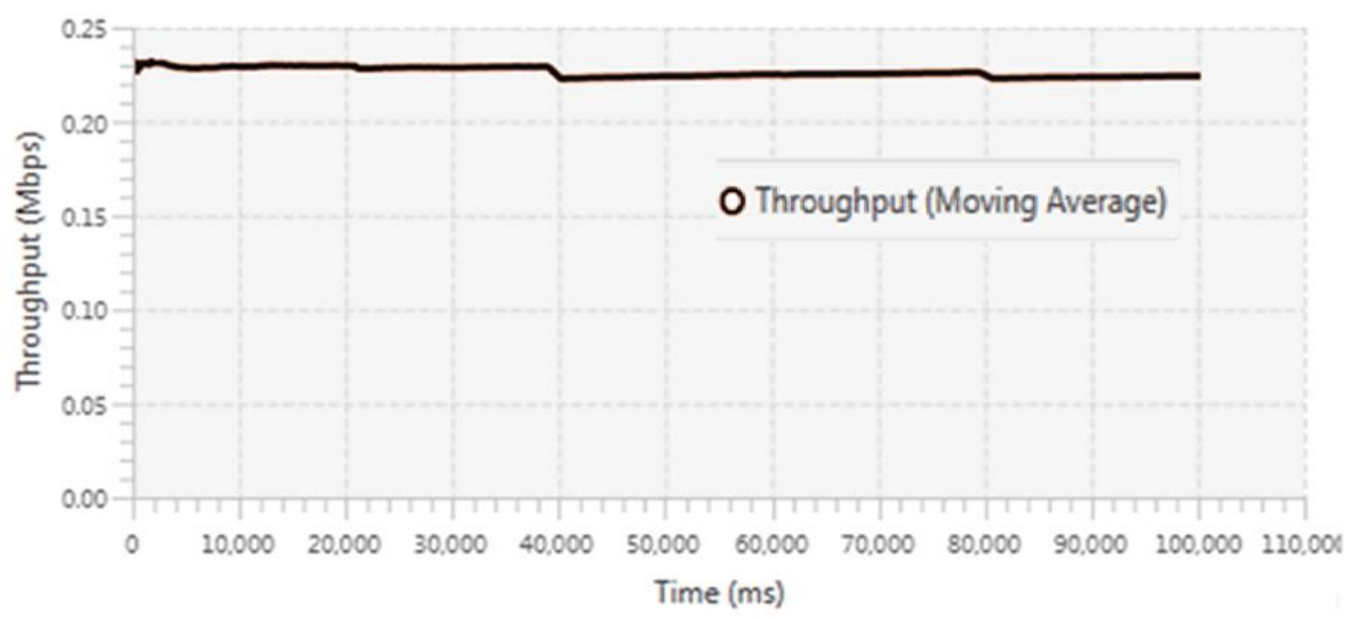

Figure 9. Scenario 3: $54 \mathrm{MHz}-802 \mathrm{MHz}$

\section{Recommendations and Future Research Direction}

A smart grid requires reliable and timely delivered sensed data to meet the expectation of various SG applications with satisfactory service delivery. The traditional or conventional SG uses probable WSN for monitoring and control in delivering the sensed data. WSN makes use of static resource allocation to statically allocate resources to the sensor node and communication devices. However, the CRSN paradigm makes use of dynamic resource allocation due to the presence of a dynamic spectrum access (DSA) capability. The CRSN paradigm works well in terms of dynamically allocating radio resources to sensor nodes and communication devices in a SG ecosystem. Hence, a CRSN makes use of dynamic resource allocation schemes to allocate resources optimally between multiple resource competitive sensor nodes.

It can be seen from the preceding section that the dynamic resource allocation schemes improve energy efficiency in the communication devices. For example, it helps to extend the battery power life of a sensor node. Unfortunately, the energy efficiency schemes in terms RRA are lacking in a CRSN based SG. Also, Table 5 shows that schemes for adaptive modulation and throughput maximization are lacking in a CRSN based SG. In addition, schemes that incorporate multiple resource optimization criteria, including a cross layer framework, as shown in Table 6, are lacking in the CRSN based SG domain.

It has been pointed out that distributed heterogeneous cluster architecture should leverage multiple improvement criteria. Thus, the authors believe that designing a holistic cross layer scheme that accommodates energy efficiency, throughput maximization and adaptive modulation, while 
leveraging multiple optimization criteria, such as interference avoidance, handoffs reduction, fairness, priority, and QoS support, etc., will go a long way in yielding optimal results in CRSN based SG monitoring and control.

Many SG applications such as distribution automation, demand response, SCADA, surveillance and multimedia applications, including security of automatic metering infrastructure (AMI), are mission critical. Hence, robust and reliable communication that can withstand harsh environmental SG conditions are required to meet the demand of these mission critical applications.

Based on this, research attention should be drawn to the direction of design and optimization of a cross layer framework for seamless exchange of signaling and control information across the protocol stack of the sensor nodes and communication devices for a CRSN based SG. It is pertinent to note that work is needed in the development of unified solution schemes that accommodate three or all of the resource optimization criteria for a CRSN based SG. Specifically, research should be directed towards energy efficient adaptive modulation, energy efficient throughput maximization, energy efficient spectrum access, and handoffs reduction. In fact, the energy efficiency issue is an open research direction in the CRSN based SG domain.

Hybrid energy harvesting that utilizes radio frequency alongside other mechanisms for harvesting energy perpetually for the power constraint sensor nodes remains an open research issue in the domain of SGs generally.

An energy efficient spectrum aware cross layer framework approach that interacts with the Physical, MAC, Routing, Transport, and Application layer protocols in CRSN based SG communication is an interesting research area.

Research should be directed towards the design of CRSNs for SG communications that will accommodate higher spectrum bands with multiple available channels from $54 \mathrm{MHz}$ to $1000 \mathrm{MHz}$ in order to support both digital TV and some 4G/LTE frequency bands.

\section{Conclusions}

In this paper, CRSN based SGs, as a new paradigm for a modern SG, has been introduced. These are different from the traditional power grid and also different from the conventional SG that uses static resource allocation techniques to allocate resources to sensor nodes and communication devices. RRA together with DSA capability to dynamically allocate radio resources to the sensor nodes and communication devices in a CRSN based SG environment has been explored.

The overview was put forward for a CRSN which introduces their unique characteristics, and functionalities. Radio resource optimization criterion, which is an important consideration for resource allocation in a CRSN based SG, has been highlighted. In addition, an improved RRA architecture called DHC architecture for a CRSN based SG [114] has been adopted in this work as a recommendation for CRSN based SG deployment. The various resource allocation schemes, i.e., RRA architecture in a CRSN based SG, have been presented in this paper. A guaranteed network connectivity channel allocation for throughput maximization (GNC-TM), including optimal spectrum band determination in RRA for improved throughput criteria in CRSNs for SGs, have been presented. The results show that the introduced model outperforms the existing protocol in terms of throughput and error probability. Recommendations have been made in order to improve communication device connectivity and seamless communication between multiple resource competitive sensor nodes in the CRSN based SG ecosystem. 
A future research direction which includes design and optimization of a cross layer framework, including new protocol architecture for RRA in a CRSN based SG, has been highlighted. Finally, energy efficiency and hybrid energy harvesting schemes for perpetual power supply to the battery power constraints sensor node have also been pointed out as open research area in a CRSN based SG.

\section{References}

1. Heggo, M.; Zhu, X.; Sumei, S.; Huang, Y. White broadband power line communication: Exploiting the TVWS for indoor multimedia smart grid applications. International Journal of Communication Systems 2017, 30, e3330.

2. Ramotsoela, D. T.; Hancke, G. P.; Abu-Mahfouz, A. M. Attack detection in water distribution systems using machine learning. Human-centric Computing and Information Sciences 2019, 9, 13.

3. Ogbodo, E. U.; Dorrell, D. G.; Abu-Mahfouz, A. M. Cognitive Radio based Sensor Network in Smart Grid: Architectures, Applications and Communication Technologies, IEEE Access 2017, 5, 19084 -19098.

4. Yu, Q.; Johnson, R. J. Smart grid communications equipment: EMI, safety, and environmental compliance testing considerations. Bell Labs Technical Journal 2011, 16, 109-131.

5. Don, H. US Smart Grid Interoperability Panel and its Testing and Certification, and Electromagnetic Interoperability Issues. In: ACIL Policy and Procedures Conference Washington, DC April 2017, 1-29.

6. Shapoury, A.; Kezunovic, M. Noise Profile of Wireless Channels in High Voltage Substations. In: Proc. IEEE Power Eng. Soc. General Meeting (PESGM ‘07), Florida, 2007.

7. Yu, Q.; Johnson, R. J. Integration of wireless communications with modernized power grids: EMI impacts and considerations. In 2011 IEEE International Symposium on Electromagnetic Compatibility 2011, 329-334.

8. Aijaz, A.; Ping, S.; Akhavan, M.; Aghvami, H. CRB-MAC: A receiver-based MAC protocol for cognitive radio equipped smart grid sensor networks. IEEE Sensors J 2014, 14, 4325-4333.

9. Shah, G. A.; Gungo, V. C.; Akan, O. B. A cross-layer design for QoS support in cognitive radio sensor networks for smart grid applications. in Proc. IEEE Int. Conf. on Communications 2012 Jun. 10-15, 13781382.

10. Shah, G. A.; Gungor, V. C.; Akan, O. B. A cross-layer QoS-aware communication framework in cognitive radio sensor networks for smart grid applications. IEEE Transactions on Industrial Informatics 2013, 9, 1477 1485.

11. Sreesha, A. A.; Somal, S.; Lu, I. T. Cognitive radio based wireless sensor network architecture for smart grid utility. in 2011 IEEE Long Island Systems, Applications and Technology Conference, 2011, 1-7.

12. Gungor, V. C.; Sahin, D. Cognitive radio networks for smart grid applications: A promising technology to overcome spectrum inefficiency. IEEE Vehic. Tech. Mag. 2012, 7, 41-46.

13. Ogbodo, E. U.; Dorrell, D. G.; Abu-Mahfouz, A. M. Performance Measurements of Communication Access Technologies and Improved CRSN Model for Smart Grid Communication, Transactions on Emerging Telecommunications and Technologies 2019, DOI: 10.1002/ett.3653.

14. Haykin, S. Cognitive radio: Brain-empowered wireless communications. IEEE J. Sel. Areas Commun. 2005, $23,201-220$.

15. Akyildiz, I. F.; Lee, W. Y.; Chowdhury, K. Spectrum management in cognitive radio ad hoc networks. IEEE Network 2009, 23, 6-12.

16. Wysocki, T.; Jamalipour, A. Spectrum management in cognitive radio: Applications of portfolio theory in wireless communications. IEEE Wireless Commun. 2011, 4, 52-60.

17. Besher, K. M.; Nieto-Hipolito, J. I.; Vazquez, B. M.; Buenrostro, M. R. SenPUI: Solutions for Sensing and Primary User Interference in Cognitive Radio Implementation of a Wireless Sensor Network. Wireless Communications and Mobile Computing. 2019.

18. Bose, A. Smart Transmission Grid Applications and Their Supporting Infrastructure. Trans. Smart Grid. 2010, $1,11-19$.

19. Farhangi, H. The Path of the Smart Grid. Power and Energy Mag. 2010, 8, 18-28.

20. Urgaonkar, R.; Neely M. J. Opportunistic scheduling with reliability guarantees in cognitive radio networks. IEEE Transactions on Mobile Computing 2009, 8, 766-777.

21. Jiang, T.; Wang, H.; Zhang, Y. Modeling channel allocation for multimedia transmission over infrastructure based cognitive radio networks. IEEE Syst. J. 2011, 5, 417-426

22. Manna, T.; Misra, I. S. 2018. A prediction and scheduling framework in centralized cognitive radio network for energy efficient non-real time communication. International Journal of Communication Systems, 2018, 31, e3716. 
1040

1041

1042

1043

1044

1045

1046

1047

1048

1049

1050

1051

1052

1053

1054

1055

1056

1057

1058

1059

1060

1061

1062

1063

1064

1065

1066

1067

1068

1069

1070

1071

1072

1073

1074

1075

1076

1077

1078

1079

1080

1081

1082

1083

1084

1085

1086

1087

1088

1089

1090

1091

1092

1093

1094

1095

23. Tragos, E. Z.; Zeadally, S.; Fragkiadakis, A. G.; Siris, V. A. Spectrum assignment in cognitive radio networks: A comprehensive survey. IEEE Commun. Surveys Tuts 2013, 15, 1108-1135.

24. Naeem, M.; Anpalagan, A.; Jaseemuddin, M.; Lee, D. C. Resource allocation techniques in cooperative cognitive radio networks. IEEE Commun. Surveys Tuts. 2014, 16, 729-744.

25. Chitnis, M.; Pagano, P.; Lipari, G.; Liang, Y. A survey on bandwidth resource allocation and scheduling in wireless sensor networks, network based information systems. in Proc. Int. Conf. NBIS, Aug 19-21, 2009, 121-128.

26. Shukry, S.; Fahmy, Y. Maximizing the lifetime of energy-constrained cooperative spectrum sensing sensor network. International Journal of Communication Systems, 2018, 31, e3569.

27. Zhang, H.; Jiang, C.; Beaulieu, N. C.; Chu, X.; Wang, X.; Quek, T. Q. Resource allocation for cognitive small cell networks: A cooperative bargaining game theoretic approach. IEEE Transactions on Wireless Communications, 2015, 14, 3481-3493

28. Zhang, H.; Nie, Y.; Cheng, J, Leung, V. C.; Nallanathan, A. Sensing time optimization and power control for energy efficient cognitive small cell with imperfect hybrid spectrum sensing. IEEE Transactions on Wireless Communications, 2017, 16, 730-743.

29. Zhang H, Jiang C, Mao X, Chen, H. H. Interference-limited resource optimization in cognitive femtocells with fairness and imperfect spectrum sensing. IEEE Transactions on Vehicular Technology, 2016, 65, 1761-1771.

30. Akkaya, K.; Younis, M. A survey on routing protocols for wireless sensor networks. Ad Hoc Netw. 2005 3, 325-349.

31. Dinu, M. A.; Ragesh, G. K. Cognitive Radio networks: A survey. In 2016 IEEE International Conference on Wireless Communications, Signal Processing and Networking (WiSPNET), Chennai, 23-25 March 2016.

32. Mendes, L.D.; Rodrigues, J. P. A survey on cross-layer solutions for wireless sensor networks. J. Netw. Comput. Appl. 2011, 34, 523-534.

33. Ahmad, A.; Ahmad, S.; Rehmani, M. H.; Hassan, N. U. A survey on radio resource allocation in cognitive radio sensor networks. IEEE Communications Surveys \& Tutorials. 2015, 17, 888-917.

34. Ireyuwa, E. I.; Oyerinde, O. O.; Viranjay, S. M.; Mneney, S. Spectrum Sensing Methodologies for Cognitive Radio Systems: A Review. International Journal of Advanced Computer Science and Applications. 2015, 6, 12.

35. Xie, R.; Yu, F. R.; Ji, H. Dynamic resource allocation for heterogeneous services in cognitive radio networks with imperfect channel sensing. IEEE transactions on vehicular technology 2012, 61, 770-80.

36. Le, L. B.; Hossain, E. Resource allocation for spectrum underlay in cognitive radio networks. IEEE Transactions on Wireless communications 2008, 7, 5306-5315.

37. Li, L.; Xu, C.; Fan, P.; He, J. Resource allocation in orthogonal frequency division multiple access-based cognitive radio systems with minimum rate constraints. International Journal of Communication Systems 2014, 27, 1147-59.

38. Xie, R.; Yu, F. R.; Ji, H.; Li, Y. Energy-efficient resource allocation for heterogeneous cognitive radio networks with femtocells. IEEE Transactions on Wireless Communications 2012, 11, 3910-3920.

39. Hosseini, E. S.; Esmaeelzadeh, V.; Berangi, R.; Akan, O. B. A correlation-based and spectrum-aware admission control mechanism for multimedia streaming in cognitive radio sensor networks. International Journal of Communication Systems 2017 30, e2986.

40. Zubair, S.; Fisal, N.; Abazeed, M.B.; Salihu, B. A.; Khan, A. S. Lightweight distributed geographical: A lightweight distributed protocol for virtual clustering in geographical forwarding cognitive radio sensor networks. International Journal of Communication Systems 2015 28, 1-18.

41. Ergul, O.; Alagoz, F.; Akan, O. B. Throughput maximization in electromagnetic energy harvesting cognitive radio sensor networks. International Journal of Communication Systems 2016, 29, 1305-1322.

42. Fadel, E.; Gungor, V. C.; Nassef, L.; Akkari, N.; Malik, M. A.; Almasri, S.; Akyildiz, I. F. A survey on wireless sensor networks for smart grid. Computer Communications 2015 71, 22-33.

43. Akan, O. B.; Karli, O.; Ergul, O. Cognitive radio sensor networks. IEEE Netw. 2009, 23, 34-40.

44. Zhao, Q.; Sadler, B. A survey of dynamic spectrum access. IEEE Signal Process. Mag. 2007, 79, 79-89.

45. Saleem, Y.; Rehmani, M. H. Primary radio user activity models for cognitive radio networks: A survey. J. Netw. Comput. Appl. 2014, 43, 1-16.

46. Varade, P. S.; Ravinder, Y. Novel Testbed implementation for resource allocation in cognitive radio for green communication In IEEE International Conference on Advances in Electronics, Communication and Computer Technology (ICAECCT), 2016, 198-203.

47. Bourdena, A.; Pallis, E.; Kormentzas, G.; Mastorakis, G. Efficient radio resource management algorithms in opportunistic cognitive radio networks. Transactions on emerging telecommunications technologies 2016, 25, 785-797. 
48. Raimundo-Neto, E.; Rosa, J. R.; Casaroli, M. A.; Feliciano da Costa, I.; Alberti, A. M.; Cerqueira Sodré, A. Implementation of an optical-wireless network with spectrum sensing and dynamic resource allocation using optically controlled reconfigurable antennas. International Journal of Antennas and Propagation, Article ID 670930, 2014.

49. Zhang, Q.; Jia, J.; Zhang, J. Cooperative relay to improve diversity in cognitive radio networks. IEEE Communications Magazine. 2009, 47, 111-117.

50. Gur, G.; Bayhan, S.; Alagoz, F. Cognitive femtocell networks: an overlay architecture for localized dynamic spectrum access [dynamic spectrum management]. IEEE Wireless Communications 2010, 17, 62-70.

51. Lu, X.; Wang, P.; Niyato, D.; Hossain, E. Dynamic spectrum access in cognitive radio networks with RF energy harvesting. IEEE Wireless Communications 2014, 21, 102-110.

52. Wen, J.; Yang, Q.; Yoo, S. J. Optimization of Cognitive Radio Secondary Information Gathering Station Positioning and Operating Channel Selection for IoT Sensor Networks. Mobile Information Systems 2018, Article ID 4721956.

53. Hu, F.; Chen, B.; Zhai, X.; Zhu, C. Channel Selection Policy in Multi-SU and Multi-PU Cognitive Radio Networks with Energy Harvesting for Internet of Everything. Mobile Information Systems 2016, Article ID 6024928.

54. Zhang, D.; Chen, Z.; Ren, J.; Zhang, N.; Awad, M. K.; Zhou, H.; Sherman, S. X. Energy-harvesting-aided spectrum sensing and data transmission in heterogeneous cognitive radio sensor network. IEEE Transactions on Vehicular Technology 2017, 66, 831-843.

55. Byun, S. S.; Balasingham, I.; Liang, X. Dynamic spectrum allocation in wireless cognitive sensor networks: Improving fairness and energy efficiency In IEEE 68th Vehicular Technology Conference, 2008, 1-5.

56. Wu, C.; Wang, Y.; Yin, Z. Energy-efficiency opportunistic spectrum allocation in cognitive wireless sensor network. EURASIP Journal on Wireless Communications and Networking 2018, 13.

57. Khan, A. A.; Rehmani, M. H.; Reisslein, M. Cognitive radio for smart grids: Survey of architectures, spectrum sensing mechanisms, and networking protocols. IEEE Communications Surveys \& Tutorials 201618 , 860-898.

58. Aroua, S. Spectrum resource assignment in cognitive radio sensor networks for smart grids. Doctoral dissertation, Université de La Rochelle, 2018.

59. Khan, Z. A.; Faheem, Y. Cognitive radio sensor networks: Smart communication for smart grids - A case study of Pakistan. Renewable and Sustainable Energy Reviews 2014, 40, 463-474.

60. Ergul, O.; Bicen, A. O.; Akan, O. B. Opportunistic reliability for cognitive radio sensor actor networks in smart grid. Ad Hoc Networks 2016 41:5-14.

61. Yu, R.; Yan, Z.; Stein, G.; Chau, Y.; Shengli, X.; Mohsen, G. Cognitive radio based hierarchical communications infrastructure for smart grid. IEEE network 2011, 25, 6-14.

62. Zhang, Y.; Yu, R.; Nekovee, M.; Liu, Y.; Shengli, X.; Stein, G. Cognitive machine-to-machine communications: visions and potentials for the smart grid. IEEE network 2012, 26, 6-13.

63. Byun, S. S.; Balasingham, I.; Xuedong, L. Dynamic spectrum allocation in wireless cognitive sensor networks: Improving fairness and energy efficiency. in IEEE 68th Vehicular Technology Conf., Sep. 21-24, 2008, 1-5.

64. Hasan, U. L.; Ejaz, W.; Atiq, M. K.; Kim, H.S.; (). Energy-efficient error coding and transmission for cognitive wireless body area network. International Journal of Communication Systems 2017, 30, e2985.

65. Zhang, H.; Zhaoyang, Z.; Chen, X.; Yin, R. Energy efficient joint source and channel sensing in cognitive radio sensor networks. in IEEE Int. Conf. on Communications 2011, 1-6.

66. Hu, Z.; Sun, Y.; Ji, Y. A dynamic spectrum access strategy based on real-time usability in cognitive radio sensor networks. in Proc. 7th Int. Conf. MSN Dec. 16-18, 2011, 318-322.

67. Gao, S.; Qian, L.; Vaman, D. R.; Qu, Q. Energy efficient adaptive modulation in wireless cognitive radio sensor networks. in Proc. IEEE Int. Conf. on Communications, Jun. 24-28, 2007, 3980-3986.

68. Ayala, J. R.; Solares, R. Z.; Alouini, M. Optimal power allocation of a single transmitter-multiple receivers channel in a cognitive sensor network. in Proc. ICWCUCA, 2012, 1-6.

69 Tao, Z.; Qin, Y.; Zhang, H.; Kuo, S. Y. A self-configurable power control algorithm for cognitive radio-based industrial wireless sensor networks with interference constraints. in Proc. IEEE Int. Conf. on Communications, 2012, 98-103.

70. Gulbahar, B.; Akan, O. B. Information theoretical optimization gains in energy adaptive data gathering and relaying in cognitive radio sensor networks. IEEE Trans. Wireless Commun. 2012, 11, 1788- 1796.

71. Askari, M.; Kavian, Y. S.; Kaabi, H.; Rashvand, H. F. A channel assignment algorithm for cognitive radio wireless sensor networks. in Proc. IET Conf. WSS, 2012, 1-4. 
1152

1153

1154

1155

1156

1157

1158

1159

1160

1161

1162

1163

1164

1165

1166

1167

1168

1169

1170

1171

1172

1173

1174

1175

1176

1177

1178

1179

1180

1181

1182

1183

1184

1185

1186

1187

1188

1189

1190

1191

1192

1193

1194

1195

1196

1197

1198

1199

1200

1201

1202

1203

1204

1205

1206

1207

1208

72. Kuo, C. H.; Chen, T. S. PN-WSNA: An approach for reconfigurable cognitive sensor network implementations. IEEE Sensors J., 2011, 11, 319-334.

73. Naeem, A.; llanko, K.; Karmokar, A.; Anpalagan, A.; Jaseemuddin, M. Energy-efficient cognitive radio sensor networks: Parametric and convex transformations. Sensors 2013, 13, 11 032-11 050.

74. Naeem, M.; Pareek, U.; Lee, D. C.; Anpalagan, A. Estimation of distribution algorithm for resource allocation in green cooperative cognitive radio sensor networks. Sensors 2013, 13, 4884-4905.

75. Han, J. A.; Jeon, W. S.; Jeong, D. G. Energy-efficient channel management scheme for cognitive radio sensor networks. IEEE Trans. Veh. Technol., 2011, 60, 1905-1910.

76. Li, X.; Wang, D.; McNair, J.; Chen, J. Residual energy aware channel assignment in cognitive radio sensor networks. in Proc. IEEE WCNC, Mar. 28-31, 2011, 398-403.

77. Li, X.; Dexiang, W.; McNair, J.; Chen, J. Dynamic spectrum access with packet size adaptation and residual energy balancing for energy constrained cognitive radio sensor networks. J. Netw. Comput. Appl. 2014, 41, 157-166.

78. Aslam, S.; Shahid, A.; Lee, K.G. Joint sensor-node selection and channel allocation scheme for cognitive radio sensor networks. J. Internet Technol. 2013, 14, 453-466.

79. Hasan, N. U.; Ejaz, W.; Lee, S.; Kim, H. S. Knapsack-based energy efficient node selection scheme for cooperative spectrum sensing in cognitive radio sensor networks. IET Commun. 2012, 6, 2998-3005.

80. Gao, S.; Qian, L.; Vaman, D. R. Distributed energy efficient spectrum access in wireless cognitive radio sensor networks. in Proc. IEEE WCNC, Mar. 31-Apr. 3, 2008, 1442-1447.

81. Lin, S.; Chen, K. Improving spectrum efficiency via in-network computations in cognitive radio sensor networks," IEEE Trans. Wireless Commun. 2014, 13, 1222-1234.

82. Liang, Z.; Feng, S.; Zhao, D.; Shen, X. Delay performance analysis for supporting real-time traffic in a cognitive radio sensor network. IEEE Trans. Wireless Commun. 2011, 10, 325-335.

83. Jamal, A.; Tham, C. K.; Wong, W. C. Event detection and channel allocation in cognitive radio sensor networks. in Proc. IEEE ICCS, 2012, 157-161.

84. Hareesh, K.; Singh, P. An energy efficient hybrid co-operative spectrum sensing technique for CRSN. in Proc. IEEE iMac4s, 2013, 438-442.

85 Shah, G.; Alagoz, F.; Fadel, E.; Akan, O. A spectrum-aware clustering for efficient multimedia routing in cognitive radio sensor networks. IEEE Trans. Veh. Technol. 2014, 63, 3369-3380.

86 Seneviratne, C.; Leung, H. A game theoretic approach for resource allocation in cognitive wireless sensor networks. in Proc. IEEE Int. Conf. SMC, Oct. 9-12, 2011 1992-1997.

87. Oto, M. C.; Akan, O. B. Energy-efficient packet size optimization for cognitive radio sensor networks. IEEE Trans. Wireless Commun. 2012 11, 1544-1553.

88. Premarathne, U. S.; Khalil, I.; Atiquzzaman, M. Secure and reliable surveillance over cognitive radio sensor networks in smart grid. Pervasive and Mobile Computing 2015, 22, 3-15.

89 Tao, Z.; Yajuan, Q.; Deyun, G.; Junqi, D.; Hongke, Z. Hybrid model design and transmission rate optimize with interference temperature constraints in cognitive radio sensor networks. in Proc. 7th Int. Conf. WiCOM, Sep. 23-25, 2011, 1-4.

90. Liu, X.; Evans, B.; Moessner, K. Energy-efficient sensor scheduling algorithm in cognitive radio networks employing heterogeneous sensors. IEEE Trans. Veh. Technol. 2013, 64, 1243 - 1249.

91. Rana, V.; Bala, I.; Jain, N. Resource allocation models for cognitive radio networks: a study. International Journal of Computer Applications 2014, 91, 51-55.

92. Lee, S.; Zhang, R.; Huang, K. Opportunistic wireless energy harvesting in cognitive radio networks," IEEE Trans. Wireless Commun. 2013, 12, 4788-4799.

93 Phuong, T. M.; Kim, D. S. Robust distributed power control for cognitive radio based industrial wireless sensor networks. Advanced Science and Technology Letters 2013, 41, 9-12.

94 Wang, Q.; Wang, J.; Lin, Y.; Tang, J.; Zhu, J. Interference management for smart grid communication under cognitive wireless network. in Proc. IEEE 3rd Int. Conf. Smart Grid Comm, Nov. 2012, 246-251.

95. Goldsmith, A. Wireless Communications, Cambridge University Press, 2005.

96. Ghorbanzadeh, M.; Abdelhadi, A.; Clancy, C. A utility proportional fairness radio resource block allocation in cellular networks. in IEEE international conference on computing, networking and communications (ICNC) 2015.

97. Yang, Y.; Zhang, Y.; Wang, Y.; Zhang, P. Average rate updating mechanism in proportional fair scheduler for HDR. IEEE Global Telecommunications Conference, Nov., 2004, 3464-3466.

98. Uchida, M.; Kurose, J. An information-theoretic characterization of weighted alpha-proportional fairness. in IEEE INFOCOM, Apr., 2009, 1053 -1061, 2009.

99. Lan, T.; Kao, D.; Chiang, M.; Sabharwal, A. An axiomatic theory of fairness. CoRR 2009, vol. abs/0906.0557. 
100. Shi, H.; Prasad, R.V.; Onur, E.; Niemegeers, I. G. Fairness in wireless networks: Issues, measures and challenges. IEEE Communications Surveys \& Tutorials 2014, 6, 5-24.

101. Hu, P.; Ibnkahla, M. A. consensus-based protocol for spectrum sharing fairness in cognitive radio ad hoc and sensor networks. in Proc. IEEE Int. Conf. on Communications, Jun. 10-15, 2012, 93-97.

102. Huang, J.; Wang, H.; Qian, Y.; Wang, C. Priority-based traffic scheduling and utility optimization for cognitive radio communication infrastructure-based smart grid. IEEE Transactions on Smart Grid 2013, 4, $78-86$.

103. Niyato, D.; Hossain, E. A game-theoretic approach to competitive spectrum sharing in cognitive radio networks. in Proc. IEEE WCNC, Hong Kong, Mar. 11-15, 2007, 16-20.

104. Izumi, S et al. (2010) A low-power multi resolution spectrum sensing (MRSS) architecture for a wireless sensor network with cognitive radio. in Proc. 4th IEEE Int. Conf. SENSORCOMM, pp 39-44, 2010.

105. Deng, R et al. Energy-efficient spectrum sensing by optimal periodic scheduling in cognitive radio networks. IET Commun. 2012, 6, 676-684.

106. Deng, R.; Chen, J.; Yuen C, Cheng, P.; Sun, Y. Energy-efficient cooperative spectrum sensing by optimal scheduling in sensor-aided cognitive radio networks. IEEE Trans. Veh. Technol. 2012, 61, 716-725.

107. Khan, Z. A.; Lehtomaki, J.; Umebayashi, K.; Vartiainen, J. On the selection of the best detection performance sensors for cognitive radio networks. IEEE Signal Process. Lett. 2010, 17, 359-362.

108. Luo, L.; Zhou, J.; Ling, P.; Roy, S.; Chen, Z.; Li, X. Heterogeneous cognitive radio sensor networks for smart grid: Markov analysis and applications. International Journal of Distributed Sensor Networks 2015, 11.

109. Tessema, N. M.; Lian, X.; Nikookar, H. Distributed beamforming with close to optimal number of nodes for green wireless sensor network. in Proc. IEEE GreenCom, 2012, 139-144.

110. Zhang, H.; Zhang, Z.; Dai, H.; Yin, R.; Chen, X. Distributed spectrum aware clustering in cognitive radio sensor networks. in Proc. IEEE GLOBECOM, 2011, 1-6.

111. Abbasi, A. A.; Younis, M. A survey on clustering algorithms for wireless sensor networks. Comput. Commun. Oct., 2007, 30, 2826- 2841.

112. Song, Y.; Zhang, C.; Fang, Y. Multiple multidimensional knapsack problem and its applications in cognitive radio networks. in Proc. MILCOM, 2008, 1-7.

113. Bicen, A. O.; Akan, O. B.; Gungor, VC. Spectrum-aware and cognitive sensor networks for smart grid applications. IEEE Commun. Mag. 2012, 50, 158-165.

114 Ogbodo, E. U.; Dorrell, D. G.; Abu-Mahfouz, A. M. Improved Resource Allocation and Network Connectivity in CRSN Based Smart Grid for Efficient Grid Automation. IEEE ICTAS, 6-8 March, Durban, South Africa, 2019.

115. Ogbodo, E. U.; Dorrell, D. G.; Abu-Mahfouz, A. M. Performance analysis of correlated multi-channels in cognitive radio sensor network based smart grid," in AFRICON, Cape Town, 1599-1604, Sept. 2017.

116. Xu, D.; Jung, E.; Liu, X. Optimal bandwidth selection in multi-channel cognitive radio networks: How much is too much? in 2008 3rd IEEE Symposium on New Frontiers in Dynamic Spectrum Access Networks, pp 111.

117. Lord, M.; Memmi, D. NetSim: a simulation and visualization software for information network modelling. in IEEE International MCETECH Conference on e-Technologies, Jan., 2008, 167-177.

118. Choudhary, A.; Tuithung, T.; Roy, O. P.; Maharaj, D. Performance evaluation of improved reliable DSR protocol in case of node failure. in IEEE Internet Technologies and Applications (ITA), 2015, 329-334. 